\title{
DEGENERATIONS OF COMPLEX DYNAMICAL SYSTEMS
}

\author{
LAURA DE MARCO ${ }^{1}$ and XANDER FABER ${ }^{2}$ \\ ${ }^{1}$ Department of Mathematics, Statistics, and Computer Science, University of Illinois at Chicago, \\ Chicago, IL, USA; \\ email: demarco@uic.edu \\ ${ }^{2}$ Department of Mathematics, University of Hawaii at Manoa, Honolulu, HI, USA; \\ email: xander@math.hawaii.edu
}

Received 28 May 2013; accepted 17 March 2014

\begin{abstract}
We show that the weak limit of the maximal measures for any degenerating sequence of rational maps on the Riemann sphere $\hat{\mathbb{C}}$ must be a countable sum of atoms. For a one-parameter family $f_{t}$ of rational maps, we refine this result by showing that the measures of maximal entropy have a unique limit on $\hat{\mathbb{C}}$ as the family degenerates. The family $f_{t}$ may be viewed as a single rational function on the Berkovich projective line $\mathbf{P}_{\mathbb{L}}^{1}$ over the completion of the field of formal Puiseux series in $t$, and the limiting measure on $\widehat{\mathbb{C}}$ is the 'residual measure' associated with the equilibrium measure on $\mathbf{P}_{\mathbb{L}}^{1}$. For the proof, we introduce a new technique for quantizing measures on the Berkovich projective line and demonstrate the uniqueness of solutions to a quantized version of the pullback formula for the equilibrium measure on $\mathbf{P}_{\mathbb{L}}^{1}$.
\end{abstract}

2010 Mathematics Subject Classification: primary 37F10, 37P50; secondary 37F45.

\section{Introduction}

Let $f_{k}: \hat{\mathbb{C}} \rightarrow \hat{\mathbb{C}}$ be a sequence of endomorphisms of the Riemann sphere of degree $d \geqslant 2$ that diverges in the space of all endomorphisms. Concretely, this means that at least one zero and pole of $f_{k}$ are colliding in the limit. Our main goal is to understand the degeneration of the dynamical features of $f_{k}$ and, ultimately, to extract useful information from a 'limit dynamical system'. In this article, we concentrate on the measure of maximal entropy.

The existence and uniqueness of a measure of maximal entropy $\mu_{f}$ for a rational function $f$ of degree $\geqslant 2$ were shown in $1983[12, \mathbf{1 5}, \mathbf{1 7}]$. Shortly after,

(c) The Author(s) 2014. The online version of this article is published within an Open Access environment subject to the conditions of the Creative Commons Attribution licence $<$ http://creativecommons.org/licenses/by/3.0/>. 
Mañé observed that the measure $\mu_{f}$ moves continuously in families [18], with the weak-* topology of measures and the uniform topology on the space of rational functions. By contrast, the Julia set $J(f)=\operatorname{supp} \mu_{f}$ fails to move continuously (in the Hausdorff topology) in the presence of bifurcations [16].

The space $\mathrm{Rat}_{d}$ of complex rational functions of degree $d \geqslant 2$ can be identified with the complement of a hypersurface in $\overline{\mathrm{Rat}}_{d}=\mathbb{P}^{2 d+1}$. In [4], the first author showed that for 'most' degenerating sequences $f_{k} \rightarrow \Phi \in \partial \mathrm{Rat}_{d}$, a limit of the maximal measures $\mu_{f_{k}}$ will exist, depending only on the limit point $\Phi$, and it can be expressed as a countably infinite sum of atoms. (The measures $\mu_{f_{k}}$ themselves are atomless.) There it was also shown that Mañé's continuity property for maximal measures does not extend to all of $\overline{\mathrm{Rat}}_{d}$. Although weak limits of maximal measures for degenerating sequences may not be unique, our first main result shows that every weak limit is purely atomic.

THEOREM A. Let $f_{k}$ be a sequence that diverges in the space Rat $_{d}$ of complex rational functions of degree $d \geqslant 2$, and assume that the measures of maximal entropy $\mu_{k}$ converge to a probability measure $\mu$ on $\hat{\mathbb{C}}$. Then $\mu$ is equal to a countable sum of atoms.

Our second main result shows that Mañé's continuity property does extend to degenerating one-parameter families. Moreover, we are able to give a refined description of the limit measure using an associated dynamical system on the Berkovich projective line.

THEOREM B. Let $\left\{f_{t}: t \in \mathbb{D}\right\}$ be a meromorphic family of rational functions of degree $d \geqslant 2$ that is degenerate at $t=0$. The measures of maximal entropy $\mu_{t}$ converge weakly on the Riemann sphere to a limiting probability measure $\mu_{0}$ as $t \rightarrow 0$. The measure $\mu_{0}$ is equal to the residual equilibrium measure for the induced rational map $f: \mathbf{P}_{\mathbb{L}}^{1} \rightarrow \mathbf{P}_{\mathbb{L}}^{1}$ on the Berkovich projective line, where $\mathbb{L}$ is the completion of the field of formal Puiseux series in $t$.

REMARK 1.1. The continuity of maximal measures on $\hat{\mathbb{C}}$ can fail for degenerating families over a parameter space of dimension two; see [4, Section 5].

REMARK 1.2. While we prefer to work with the more 'geometric' field $\mathbb{L}$, one can replace it with the field of formal Laurent series $\mathbb{C}((t))$ in the statement of the theorem.

One should view the Berkovich dynamical system $\left(f, \mathbf{P}_{\mathbb{L}}^{1}\right)$ as the limit of dynamical systems $\left(f_{t}, \hat{\mathbb{C}}\right)$ as $t \rightarrow 0$. This fruitful perspective was introduced 
by Jan Kiwi in his work on cubic polynomials and quadratic rational maps; see $[13,14]$ and [3]. A closely related construction, viewing degenerations of polynomial maps as actions on trees, can be seen in [7]. Charles Favre has recently constructed a compactification of the space of rational maps, where the boundary points are rational maps on a Berkovich $\mathbf{P}^{1}$ [10]. Our work is very much inspired by these results. The Berkovich space viewpoint allows us to recover the results in [4], and it provides a conceptual explanation for the form of the limiting measures. In a sequel to this article, we describe a countable-state Markov process that allows one to compute the residual measure explicitly [6].

As with nondegenerating families, the Julia sets of $f_{t}$ may fail to converge to a limit as $t \rightarrow 0$. Consider the example of $f_{t}(z)=t\left(z+z^{-1}\right)$ in $\mathrm{Rat}_{2}$. As $t \rightarrow 0$ along the real axis, the Julia set of $f_{t}$ is equal to the imaginary axis, while there is a sequence $t_{n} \rightarrow 0$ (tangent to the imaginary axis) for which $J\left(f_{t_{n}}\right)=\hat{\mathbb{C}}$. Mañé used the continuity of $f \mapsto \mu_{f}$ to deduce that the Hausdorff dimension of $\mu_{f}$ is a continuous function of $f$, but this property does not extend to degenerating families; for example, the measures for a flexible Lattès family have dimension two, while the limit measures always have dimension zero.

The measure of maximal entropy $\mu_{f}$ for a rational function $f$ of degree $d \geqslant 2$ is characterized by the conditions that (a) it does not charge exceptional points, and (b) it satisfies the pullback relation

$$
\frac{1}{d} f^{*} \mu_{f}=\mu_{f}
$$

To prove Theorem A, we show that any weak limit of measures of maximal entropy on $\hat{\mathbb{C}}$ must satisfy an appropriately defined pullback formula (Theorem 2.4); we then show that any measure satisfying this formula (for all iterates) is atomic. The pullback formula is phrased in terms of 'paired measures', which are ad hoc objects that we introduce to keep track of weak limits of measures in two sets of coordinates simultaneously. This is all accomplished in Section 2.

The proof of Theorem B (which inspired our proof of Theorem A) is more conceptual and can be divided into three parts, each with its own collection of results that are of independent interest. We sketch these results here.

Step 1. Dynamics on a complex surface. In Section 3, we view the holomorphic family $f_{t}: \mathbb{P}^{1} \rightarrow \mathbb{P}^{1}$ as one (meromorphic) dynamical system

$$
F: X \rightarrow X
$$

on the complex surface $X=\mathbb{D} \times \mathbb{P}^{1}$, given by $(t, z) \mapsto\left(t, f_{t}(z)\right)$ for $t \neq 0$. By hypothesis, $F$ will have points of indeterminacy in the central fiber $X_{0}=\{0\} \times \mathbb{P}^{1}$. 
If $F$ collapses $X_{0}$ to a point, we let $\pi: Y \rightarrow X$ be the (minimal) blow-up of the target surface such that $F: X \rightarrow Y$ is nonconstant at $t=0$; otherwise, set $Y=X$ and $\pi=\mathrm{Id}$. By counting multiplicities at the indeterminacy points of $F$, we define a notion of pullback $F^{*}$ from measures on the central fiber of $Y$ to measures on $X_{0}$. We prove (Theorem 3.5) that any weak limit $\nu$ of the measures $\mu_{t}$ on the central fiber of $Y$ satisfies a pullback relation:

$$
\frac{1}{d} F^{*} \nu=\pi_{*} \nu
$$

The proof relies on the Argument Principle for handling the measure at the points of indeterminacy for $F$.

Step 2. Dynamics and $\boldsymbol{\Gamma}$-measures on the Berkovich projective line. Let $k$ be an algebraically closed field of characteristic zero that is complete with respect to a nontrivial non-Archimedean absolute value. The Berkovich analytification of the projective line $\mathbb{P}_{k}^{1}$ will be denoted as $\mathbf{P}^{1}$; it is a compact, Hausdorff, and uniquely arcwise connected topological space. A rational function $f: \mathbb{P}_{k}^{1} \rightarrow \mathbb{P}_{k}^{1}$ extends functorially to $\mathbf{P}^{1}$. If $d=\operatorname{deg}(f) \geqslant 2$, then the equilibrium measure $\mu_{f}$ may be characterized as in the complex case by the conditions that (a) it does not charge exceptional points of $\mathbb{P}^{1}(k)$, and (b) it satisfies the pullback relation $\frac{1}{d} f^{*} \mu_{f}=\mu_{f}$ [11]. See [1] for a reference specific to the dynamics on $\mathbf{P}^{1}$, or see [2] for the more general theory of non-Archimedean analytic spaces.

The goal of Section 4 is to define a notion of pullback $f^{*}$ on a new space of quantized measures relative to a finite set $\Gamma$ of vertices in $\mathbf{P}^{1}$. Every Borel probability measure $v$ on $\mathbf{P}^{1}$ gives rise to one of these ' $\Gamma$-measures' $v_{\Gamma}$. And if $v$ is a solution to the standard pullback formula $\frac{1}{d} f^{*} v=v$, then $v_{\Gamma}$ will satisfy a quantized version:

$$
\frac{1}{d} f^{*} v_{\Gamma}=\pi_{*} v_{\Gamma}
$$

(One must push $v_{\Gamma}$ forward by a certain map $\pi$ in order to have a meaningful equation since $f^{*} v_{\Gamma}$ lies in the space of $\Gamma^{\prime}$-measures for a potentially different vertex set $\Gamma^{\prime}$.) A solution to the pullback formula (1.0.2) is typically far from unique. However, we will show (Theorem 4.10) that uniqueness is restored if one considers simultaneous solutions to pullback equations for all iterates of $f$, after ruling out measures supported on classical exceptional cycles.

Step 3. A transfer principle. Now, let $k=\mathbb{L}$ be the completion of the field of formal Puiseux series in $t$, equipped with the non-Archimedean absolute value that measures the order of vanishing at $t=0$. (See [14, Section 3].) On viewing the parameter $t$ as an element of $\mathbb{L}$, the family $f_{t}$ defines a single rational function $f$ with coefficients in $\mathbb{L}$. We define a vertex set $\Gamma \subset \mathbf{P}^{1}$ consisting of one 
vertex only, the Gauss point. In Section 5.2, we define a correspondence between measures on the central fiber of our surface $X$ with $\Gamma$-measures on $\mathbf{P}^{1}$. From Step 1, any weak limit $v$ of the measures $\mu_{t}$ will satisfy the pullback relation (1.0.1). The corresponding $\Gamma$-measure $v_{\Gamma}$ must satisfy the non-Archimedean pullback relation (1.0.2) on $\mathbf{P}^{1}$, by Proposition 5.1. We repeat the argument for all iterates $f_{t}^{n}$. From Step 2, we deduce that $v_{\Gamma}$ is the equilibrium $\Gamma$-measure, and consequently, the limit measure $v$ is the 'residual' equilibrium measure. See Section 5.

\section{The space of rational maps: complex-analytic arguments}

In this section we prove Theorem A along with a number of preliminary results that will be used in the first step of the proof of Theorem B. In [4], the first author obtained a version of Theorem A under an additional hypothesis. The observations of Lemma 2.1 and (the more refined result in) Lemma 2.5 concerning Möbius rescalings allow us to obtain the complete statement. These lemmas were inspired by the Berkovich space structure appearing in the proof of Theorem B.

2.1. The space of rational maps. We will let $\mathrm{Rat}_{d}$ denote the set of all complex rational functions of degree $d$. It can be viewed as an open subset of the complex projective space $\mathbb{P}^{2 d+1}$, by identifying a function

$$
f(z)=\frac{a_{0} z^{d}+a_{1} z^{d-1}+\cdots+a_{d}}{b_{0} z^{d}+b_{1} z^{d-1}+\cdots+b_{d}}
$$

with its coefficients in homogeneous coordinates

$$
\left(a_{0}: a_{1}: \cdots: a_{d}: b_{0}: b_{1}: \cdots: b_{d}\right) \in \mathbb{P}^{2 d+1} .
$$

In fact, any point $\Phi \in \mathbb{P}^{2 d+1}$ determines a pair $(P, Q)$ of homogeneous polynomials in two variables, and $\operatorname{Rat}_{d}=\mathbb{P}^{2 d+1} \backslash\{\operatorname{Res}(P, Q)=0\}$. We set $\overrightarrow{\operatorname{Rat}}_{d}=\mathbb{P}^{2 d+1}$ so that $\partial \mathrm{Rat}_{d}=\{$ Res $=0\}$. For each $\Phi=(P, Q) \in \partial \mathrm{Rat}_{d}$, we let $H=\operatorname{gcd}(P, Q)$, and let $\phi$ be the induced rational function of degree $<d$ defined by the ratio $P / Q$. To match the algebraic language of the later sections, we refer to the map $\phi$ as the reduction of $\Phi$.

A one-parameter holomorphic family $\left\{f_{t}: t \in U\right\}$ is a holomorphic map from a domain $U \subset \mathbb{C}$ to $\mathrm{Rat}_{d}$. A meromorphic family is a holomorphic map from $U$ to $\overline{\mathrm{Rat}}_{d}$ with image not contained in $\partial \mathrm{Rat}_{d}$. A meromorphic family is degenerate at $u \in U$ if the image of $u$ lies in $\partial \operatorname{Rat}_{d}$.

LEMMA 2.1. Let $f_{k}$ be a sequence in Rat $_{d}$ converging to a point $\Phi \in \partial$ Rat $_{d}$. After passing to a subsequence if necessary, there is a sequence of Möbius 
transformations $A_{k} \in \mathrm{Rat}_{1}$ such that $A_{k} \circ f_{k}$ converges in $\overline{\mathrm{Rat}}_{d}$ to a point with nonconstant reduction. If $B_{k}$ is any other such sequence in $\mathrm{Rat}_{1}$, then $M_{k}=$ $A_{k} \circ B_{k}^{-1}$ converges in $\mathrm{Rat}_{1}$ as $k \rightarrow \infty$ (along the subsequence determined by $\left.A_{k}\right)$. If the $f_{k}$ lie in a meromorphic family $\left\{f_{t}: t \in \mathbb{D}\right\}$, then the sequence $A_{k}$ may be chosen to lie in a meromorphic family $\left\{A_{t}: t \in \mathbb{D}\right\}$.

Proof. The proof of existence is carried out, algorithmically, in [19, Prop. 2.4] and appears also in [14, Lemma 3.7] for when the sequence lies in a holomorphic family; the strategy is as follows.

At each step of this argument, we may pass to a subsequence. Write

$$
f_{k}(z, w)=\left(P_{k}(z, w): Q_{k}(z, w)\right)
$$

normalized such that $\left(P_{k}, Q_{k}\right) \rightarrow(P, Q)$ in $\overline{\operatorname{Rat}}_{d}$. Note that at least one of $P$ and $Q$ is nonzero. By replacing $f_{k}$ with $S_{k} \circ f_{k}$, where $S_{k}(z)=\alpha_{k} z$ with $\alpha_{k}>0$, it can be arranged that the limiting $P$ and $Q$ are both nonzero. If $P$ is not a scalar multiple of $Q$, we are done.

Suppose $P=c_{0} Q$ for some constant $c_{0} \in \mathbb{C}^{*}$. If $m=\operatorname{deg}_{z} P=\operatorname{deg}_{z} Q$, write

$$
f_{k}(z, w)=\left(a_{k} z^{m} w^{d-m}+\hat{P}_{k}(z, w): b_{k} z^{m} w^{d-m}+\hat{Q}_{k}(z, w)\right)
$$

where $\hat{P}_{k}$ and $\hat{Q}_{k}$ have no term involving $z^{m} w^{d-m}$. Now, postcompose $f_{k}$ with a translation by $a_{k} / b_{k}=c_{0}+o(1)$, replacing $f_{k}$ with

$$
f_{k}(z, w)=\left(P_{k}(z, w)-a_{k} b_{k}^{-1} Q_{k}(z, w): Q_{k}(z, w)\right) .
$$

If $P$ and $Q$ are not monomials, then we are done; the new limit has nonconstant reduction. If $P$ and $Q$ are monomials, the resulting limit in $\overline{\mathrm{Rat}}_{d}$ will have constant reduction $(=0)$; we rescale and repeat the initial argument. It follows that the new $P$ cannot be a scalar multiple of $Q$ because it has no term involving $z^{m} w^{d-m}$. This completes the proof of existence of $\left\{A_{k}\right\}$.

If the given $f_{k}$ lies in a meromorphic family $f_{t}=\left(P_{t}, Q_{t}\right)$, then the scaling and translation maps can be chosen meromorphic in $t$, since they are built from the coefficients of $f_{t}$.

Now suppose that $A_{k} \circ f_{k} \rightarrow \Phi_{A}$ and $B_{k} \circ f_{k} \rightarrow \Phi_{B}$ in $\overline{\text { Rat }}_{d}$, with nonconstant reductions $\phi_{A}$ and $\phi_{B}$. Set $M_{k}=A_{k} \circ B_{k}^{-1}$. Again passing to a subsequence, $M_{k}$ converges to $M_{0} \in \overline{\mathrm{Rat}}_{1}$. Away from finitely many points in $\mathbb{P}^{1}$, we have

$$
\phi_{A}(p)=\lim _{k \rightarrow \infty} A_{k} \circ f_{k}(p)=\lim _{k \rightarrow \infty} M_{k} \circ B_{k} \circ f_{k}(p)=M_{0} \circ \phi_{B}(p) .
$$

As $\phi_{A}$ is nonconstant, so is $M_{0}$, and therefore $M_{0} \in \mathrm{Rat}_{1}$. This also shows that $M_{0}$ is uniquely determined, so the full sequence $M_{k}$ converges. 
2.2. Counting preimages. Fix a sequence $f_{k}$ in Rat ${ }_{d}$, and assume that $f_{k}$ converges to a degenerate point $\Phi \in \partial \mathrm{Rat}_{d}$ with gcd $H$ and nonconstant reduction $\phi$. For each point $x \in \mathbb{P}^{1}$, we define multiplicities

$$
m(x)=\operatorname{deg}_{x} \phi \quad \text { and } \quad s(x)=\operatorname{ord}_{x} H .
$$

The quantity $m(x)$ is the local degree of $\phi$, and the quantity $s(x)$ will be called the surplus multiplicity at $x$.

Let $\eta$ be a small loop around $\phi(x)$ bounding a disk $D$, and let $\gamma_{x}$ be the small loop around $x$ sent with degree $m(x)$ onto $\eta$ by $\phi$. Choose $\gamma_{x}$ small enough that it does not contain any roots of $H$, except possibly $x$ itself. Because $f_{k}$ converges locally uniformly to $\phi$ on $\mathbb{P}^{1} \backslash\{H=0\}$, for each $k \gg 0$ there is a small loop $\gamma_{k}$ around $x$ that is mapped by $f_{k}$ with degree $m(x)$ onto $\eta$. Let $U_{k}$ be the domain bounded by $\gamma_{k}$.

Proposition 2.2. Assume that $f_{k}$ converges to $\Phi \in \partial$ Rat $_{d}$ with nonconstant reduction. Fix $x \in \mathbb{P}^{1}$. For all $k$ sufficiently large,

$$
\#\left(f_{k}^{-1}\left(z_{0}\right) \cap \bar{U}_{k}\right)=m(x)+s(x)
$$

and

$$
\#\left(f_{k}^{-1}\left(p_{0}\right) \cap \bar{U}_{k}\right)=s(x),
$$

for all points $z_{0}$ in $\bar{D}$ and all points $p_{0}$ in $\mathbb{P}^{1} \backslash \bar{D}$.

Proof. The proof is an application of the Argument Principle from complex analysis. Assume first that $z_{0}=0 \in D$ and $p_{0}=\infty \notin \bar{D}$. Then

$$
\#\left(f_{k}^{-1}\left(z_{0}\right) \cap U_{k}\right)=\#\left(f_{k}^{-1}\left(z_{0}\right) \cap \bar{U}_{k}\right)=\# \operatorname{Zeroes}\left(f_{k}\right) \text { inside } U_{k},
$$

and

$$
\#\left(f_{k}^{-1}\left(p_{0}\right) \cap U_{k}\right)=\#\left(f_{k}^{-1}\left(p_{0}\right) \cap \bar{U}_{k}\right)=\# \operatorname{Poles}\left(f_{k}\right) \text { inside } U_{k} .
$$

By the Argument Principle, for all large $k$ we have

$$
\#\left(f_{k}^{-1}\left(z_{0}\right) \cap U_{k}\right)-\#\left(f_{k}^{-1}\left(p_{0}\right) \cap U_{k}\right)=\int_{\gamma_{k}} \frac{f_{k}^{\prime}}{f_{k}}=m(x) .
$$

On the other hand, we may compute directly that

$$
s(x)=\# \operatorname{Poles}\left(f_{k}\right) \text { inside } U_{k}
$$

for all sufficiently large $k$, since $f_{k} \rightarrow \Phi$. Indeed, $H(x)=0$ with multiplicity $s(x)$ (and $\phi(x) \neq \infty$ ), so there are exactly $s(x)$ poles converging to $x$ as $k \rightarrow \infty$. (Compare [4, Lemma 4.1].) It remains to handle the case where $z_{0} \in \eta=\partial D$. By construction, the boundary $\gamma_{k}$ of $U_{k}$ is mapped with degree $m(x)$ over $\eta$; and by viewing $z_{0}$ as the point $\infty$, we see that there must be $s(x)$ preimages of $z_{0}$ converging to $x$ as $k \rightarrow \infty$. 
2.3. Pullback by a degenerate map. Let $\Phi$ be an element of $\overline{\mathrm{Rat}}_{d}$ with nonconstant reduction $\phi$. Exactly as in [4, Section 3], we define the pullback of a measure $\mu$ on $\mathbb{P}^{1}$ by the formula

$$
\Phi^{*} \mu:=\phi^{*} \mu+\sum_{x \in \mathbb{P}^{1}} s(x) \delta_{x} .
$$

Recall that $s(x)$ is defined in (2.2.1).

LEMMA 2.3. For any probability measure $\mu$ and $\Phi \in \overline{\mathrm{Rat}}_{d}$ with nonconstant reduction, the measure $\Phi^{*} \mu$ has total mass $d$.

Proof. The proof is a simple degree count:

$$
\Phi^{*} \mu\left(\mathbb{P}^{1}\right)=\operatorname{deg}(\phi)+\sum_{x \in \mathbb{P}^{1}} s(x)=\operatorname{deg}(\phi)+\operatorname{deg}(H)=d .
$$

2.4. Paired measures and weak limits. Let $C, E$ denote two copies of $\mathbb{P}^{1}$. A paired measure $\left(\mu_{C}, \mu_{E}\right)$ is a pair of Borel probability measures $\mu_{C}$ on $C$ and $\mu_{E}$ on $E$. Let $\left\{A_{k}\right\}$ be a sequence of Möbius transformations in Rat ${ }_{1}$. We say that a sequence of Borel probability measures $\left\{\mu_{k}\right\}$ on $\mathbb{P}^{1}$ converges $\left\{A_{k}\right\}$-weakly to the paired measure $\left(\mu_{C}, \mu_{E}\right)$ if

$$
\mu_{k} \rightarrow \mu_{C} \quad \text { and } \quad A_{k *} \mu_{k} \rightarrow \mu_{E}
$$

weakly. Note that, if $A_{k} \rightarrow A \in \mathrm{Rat}_{1}$, then necessarily $A_{*} \mu_{C}=\mu_{E}$. It will often be the case that $A_{k}$ diverges in $\mathrm{Rat}_{1}$, as in Lemma 2.1. In Section 3, $C$ and $E$ will represent components of the 'central fiber' of a complex surface.

THEOREM 2.4. Let $\left\{f_{k}\right\}$ be a sequence in $\operatorname{Rat}_{d}$ and $\left\{A_{k}\right\}$ a sequence in $\mathrm{Rat}_{1}$ such that $A_{k} \circ f_{k}$ converges to $\Phi \in \overline{\mathrm{Rat}}_{d}$ with nonconstant reduction. Any $\left\{A_{k}\right\}$-weak limit $\left(\mu_{C}, \mu_{E}\right)$ of the maximal measures $\mu_{f_{k}}$ will satisfy the pullback formula

$$
\frac{1}{d} \Phi^{*} \mu_{E}=\mu_{C}
$$

Proof. Without loss of generality, we may replace $f_{k}$ with a subsequence in order to allow the assumption that $\mu_{f_{k}}$ converges $\left\{A_{k}\right\}$-weakly to $\left(\mu_{C}, \mu_{E}\right)$. For simplicity, we will write $\mu_{k}$ for $\mu_{f_{k}}$. By the definition of $\left\{A_{k}\right\}$-weak convergence, and because $d^{-1} f_{k}^{*} \mu_{k}=\mu_{k}$ for all $k$, we know that

$$
d^{-1} f_{k}^{*} \mu_{k} \rightarrow \mu_{C} \quad \text { as } \quad k \rightarrow \infty .
$$

We need to show that the weak limit of $f_{k}^{*} \mu_{k}$ can also be expressed as $\Phi^{*} \mu_{E}$. 
Let $H$ be the gcd of $\Phi$, and let $\phi$ be the reduction map of degree $>0$. Let $I(\Phi)$ denote the union of the roots of $H$. Let $U$ be a small neighborhood of $I(\Phi)$ in $\mathbb{P}^{1}$. Choose a partition of unity

$$
b_{r}+b_{s} \equiv 1,
$$

subordinate to the open cover $\left\{\mathbb{P}^{1} \backslash I(\Phi), U\right\}$ such that $b_{r} \equiv 1$ on $\mathbb{P}^{1} \backslash U$ and $b_{s} \equiv$ 1 on a small neighborhood of $I(\Phi)$ inside $U$; as usual, $b_{r}$ and $b_{s}$ are nonnegative continuous functions.

Fix a nonnegative continuous function $\psi$ on $\mathbb{P}^{1}$. Recall that the pushforward of $\psi$ by $f \in$ Rat $_{d}$ can be defined by

$$
f_{*} \psi(y)=\sum_{f(x)=y} \psi(x),
$$

where preimages are counted with multiplicity. Because $b_{r}$ vanishes near $I(\Phi)$, and because $A_{k} \circ f_{k}$ converges uniformly to $\phi$ on compact sets outside $I(\Phi)$, we have uniform convergence of functions

$$
\left(A_{k} \circ f_{k}\right)_{*}\left(b_{r} \psi\right) \rightarrow \phi_{*}\left(b_{r} \psi\right),
$$

and therefore

$$
\begin{aligned}
\int b_{r} \psi\left(f_{k}^{*} \mu_{k}\right) & =\int b_{r} \psi\left(\left(A_{k} \circ f_{k}\right)^{*} A_{k *} \mu_{k}\right) \\
& =\int\left(A_{k} \circ f_{k}\right)_{*}\left(b_{r} \psi\right) A_{k *} \mu_{k} \rightarrow \int \phi_{*}\left(b_{r} \psi\right) \mu_{E} \\
& =\int b_{r} \psi \Phi^{*} \mu_{E},
\end{aligned}
$$

by the weak convergence of $A_{k *} \mu_{k}$ to $\mu_{E}$. Upon shrinking the neighborhood $U$, (2.4.1) and (2.4.2) together will show that

$$
\int_{\mathbb{P}^{1} \backslash I(\Phi)} \psi \mu_{C}=\frac{1}{d} \int_{\mathbb{P}^{1} \backslash I(\Phi)} \psi \Phi^{*} \mu_{E}
$$

for any test function $\psi$.

Fix $x \in I(\Phi)$. As in Section 2.2, let $\eta$ be a small loop around $\phi(x)$ that bounds an open disk $D$, and let $\gamma_{x}$ be the small loop around $x$ sent with degree $m(x)$ onto $\eta$ by $\phi$. Choose $\gamma_{x}$ small enough that it does not contain any point in $I(\Phi)$ other than $x$ itself; we shall further assume that it is contained in the neighborhood where $b_{s} \equiv 1$. Because $A_{k} \circ f_{k}$ converges locally uniformly to $\phi$ on $\mathbb{P}^{1} \backslash I(\Phi)$, for each $k \gg 0$ there is a small loop $\gamma_{k}$ around $x$ that is mapped by $f_{k}$ with degree $m(x)$ onto $\eta$; for large $k$, this $\gamma_{k}$ is also contained in the region where $b_{s} \equiv 1$. Let $U_{x, k}$ be the domain bounded by $\gamma_{k}$. 
We now apply Proposition 2.2 to the sequence $A_{k} \circ f_{k}$. For $x \in I(\Phi)$, let $\psi_{\text {inf }}(x)$ denote the infimum of $\psi$ on the component of $U$ containing $x$. For all $k$ sufficiently large,

$$
\begin{aligned}
\int_{\mathbb{P}^{1}} b_{s} \psi\left(f_{k}^{*} \mu_{k}\right) & \geqslant \sum_{x \in I(\Phi)} \psi_{\mathrm{inf}}(x) \int_{\bar{U}_{x, k}}\left(A_{k} \circ f_{k}\right)^{*} A_{k *} \mu_{k} \\
& =\sum_{x \in I(\Phi)} \psi_{\mathrm{inf}}(x) \int_{\mathbb{P}^{1}} \#\left(\left(A_{k} \circ f_{k}\right)^{-1}(y) \cap \bar{U}_{x, k}\right) A_{k *} \mu_{k}(y) \\
& =\sum_{x \in I(\Phi)} \psi_{\mathrm{inf}}(x)\left[s(x) A_{k *} \mu_{k}\left(\mathbb{P}^{1} \backslash \bar{D}\right)+(m(x)+s(x)) A_{k *} \mu_{k}(\bar{D})\right] \\
& =\sum_{x \in I(\Phi)} \psi_{\mathrm{inf}}(x)\left[s(x)+m(x) A_{k *} \mu_{k}(\bar{D})\right] .
\end{aligned}
$$

Letting $k \rightarrow \infty$, the $\left\{A_{k}\right\}$-weak convergence of measures gives

$$
\liminf _{k \rightarrow \infty} A_{k *} \mu_{k}(\bar{D}) \geqslant \mu_{E}(\{\phi(x)\}) .
$$

Because $d^{-1} f_{k}^{*} \mu_{k}$ converges weakly to $\mu_{C}$, we deduce that

$$
\int b_{s} \psi \mu_{C} \geqslant \frac{1}{d} \sum_{x \in I(\Phi)}\left[s(x)+m(x) \mu_{E}(\{\phi(x)\})\right] \psi_{\min }(x) .
$$

Shrinking the neighborhood $U$ of $I(\Phi)$, we obtain

$$
\int_{I(\Phi)} \psi \mu_{C} \geqslant \frac{1}{d} \sum_{x \in I(\Phi)}\left[s(x)+m(x) \mu_{E}(\{\phi(x)\})\right] \psi(x)=\frac{1}{d} \int_{I(\Phi)} \psi \Phi^{*} \mu_{E} .
$$

As $\psi$ was arbitrary, adding (2.4.3) to (2.4.4) yields the inequality of positive measures

$$
\mu_{C} \geqslant \frac{1}{d} \Phi^{*} \mu_{E}
$$

But both are probability measures (by Lemma 2.3), so we must have equality.

2.5. Proof of Theorem A. Let $f_{k}$ be a sequence in Rat ${ }_{d}$ converging to $f_{0} \in \partial \mathrm{Rat}_{d}$ and with maximal measures $\mu_{k}$ converging to a measure $\mu$. From Lemma 2.1, there is a sequence $A_{k} \in \mathrm{Rat}_{1}$ such that $A_{k} \circ f_{k}$ converges to $\Phi \in \overline{\mathrm{Rat}}_{d}$ with reduction $\phi$ of positive degree. Passing to subsequences for each iterate $n$ and applying a diagonalization argument, we choose sequences $\left\{A_{n, k}: k \in \mathbb{N}\right\}$ in $\mathrm{Rat}_{1}$ such that

$$
A_{n, k} \circ f_{k}^{n} \rightarrow \Phi_{n} \quad \text { as } k \rightarrow \infty
$$


in $\overline{\mathrm{Rat}}_{d^{n}}$ with reduction $\phi_{n}$ such that $\operatorname{deg} \phi_{n}>0$ for every iterate $n$. By sequential compactness of the space of probability measures on $\mathbb{P}^{1}$ (and another diagonalization argument, if necessary), we may assume that $\mu_{k}$ converges $\left\{A_{n, k}\right\}$ weakly to a paired measure $\left(\mu, \mu_{E_{n}}\right)$ as $k \rightarrow \infty$ for each $n \geqslant 1$.

Since the measures $\mu_{k}$ are also the measures of maximal entropy for iterates $f_{k}^{n}$, Theorem 2.4 implies that

$$
\mu(\{p\})=\frac{1}{d^{n}} \Phi_{n}^{*} \mu_{E_{n}}(\{p\}) \geqslant \frac{s_{\Phi_{n}}(p)}{d^{n}}
$$

for any iterate $n$ and any point $p \in \mathbb{P}^{1}$; recall that the integers $s_{\Phi_{n}}(p)$ are defined in (2.2.1). Degree counting shows that $\sum_{p \in \mathbb{P}^{1}} S_{\Phi_{n}}(p)=d^{n}-\operatorname{deg} \phi_{n}$, which yields

$$
1 \geqslant \sum_{p \in \mathbb{P}^{1}} \mu(\{p\}) \geqslant 1-\frac{\operatorname{deg} \phi_{n}}{d^{n}} .
$$

If $\operatorname{deg} \phi_{n}=o\left(d^{n}\right)$ as $n \rightarrow \infty$, then we see immediately that $\mu$ is a countable sum of atoms. It remains to treat the case where $\operatorname{deg} \phi_{n} \neq o\left(d^{n}\right)$.

The next lemma shows that the reduction maps $\phi_{n}$ are not unrelated.

LEMMA 2.5. The reduction maps $\phi_{n}$ form a composition sequence. That is, there exist rational functions $\phi_{n+1, n}$ of positive degrees $\leqslant d$ such that

$$
\phi_{n+1}=\phi_{n+1, n} \circ \phi_{n}
$$

for each $n \geqslant 1$. Moreover, $A_{n+1, k} \circ f_{k} \circ A_{n, k}^{-1}$ converges to $\phi_{n+1, n}$ away from finitely many points in $\mathbb{P}^{1}$.

Proof. This lemma follows from uniqueness in Lemma 2.1. Write $\Phi=H \phi$ for any $\Phi \in \overline{\mathrm{Rat}}_{d}$, where $H$ is the gcd of the two polynomials defining $\Phi$ and $\phi$ is the reduction. As $k \rightarrow \infty$, we have $A_{n, k} \circ f_{k}^{n} \rightarrow H_{n} \phi_{n}$ and $A_{n+1, k} \circ f_{k}^{n+1} \rightarrow H_{n+1} \phi_{n+1}$. Consider the sequence $f_{k} \circ A_{n, k}^{-1}$ in $\mathrm{Rat}_{d}$. Passing to a subsequence, there exists a sequence $C_{k}$ of Möbius transformations such that $C_{k} \circ f_{k} \circ A_{n, k}^{-1} \rightarrow H \phi$ with $\operatorname{deg} \phi>0$. But then, by the continuity of degenerate composition (exactly as in [5, Lemma 2.6]), we have

$$
\left(C_{k} \circ f_{k} \circ A_{n, k}^{-1}\right) \circ\left(A_{n, k} \circ f_{k}^{n}\right)=C_{k} \circ f_{k}^{n+1} \rightarrow\left(H_{n}^{d} \cdot\left(H \circ \phi_{n}\right)\right) \phi \circ \phi_{n} .
$$

But uniqueness in Lemma 2.1 then implies that there exists a Möbius transformation $B=\lim _{k \rightarrow \infty} A_{n+1, k} \circ C_{k}^{-1}$ such that $\phi_{n+1}=B \circ \phi \circ \phi_{n}$. We set $\phi_{n+1, n}=B \circ \phi$. 
Lemma 2.5 implies that the degree of $\phi_{n}$ may be computed by using

$$
\operatorname{deg} \phi_{n}=\operatorname{deg} \phi_{1} \cdot \prod_{j=1}^{n-1} \operatorname{deg} \phi_{j+1, j}
$$

In particular, $\operatorname{deg} \phi_{n} \neq o\left(d^{n}\right)$ implies that there exists $n_{0}>0$ such that $\operatorname{deg} \phi_{n+1, n}=d$ for all $n \geqslant n_{0}$. For the remainder of the proof, we will operate under this assumption.

Suppose for the moment that there exist nonnegative integers $m>n \geqslant n_{0}$ such that

$$
A_{n, k} \circ A_{m, k}^{-1} \rightarrow L \in \mathrm{Rat}_{1} \quad \text { as } k \rightarrow \infty
$$

(after passing to a subsequence, if necessary). From Lemma 2.5 and the continuity of composition,

$$
A_{n, k} \circ f_{k}^{m-n} \circ A_{n, k}^{-1}=A_{n, k} \circ A_{m, k}^{-1} \circ A_{m, k} \circ f_{k}^{m-n} \circ A_{n, k}^{-1} \longrightarrow L \circ \phi_{m, m-1} \circ \cdots \circ \phi_{n+1, n},
$$

and the limiting function has degree $d^{m-n}$. In other words, the sequence of conjugates $A_{n, k} \circ f_{k}^{m-n} \circ A_{n, k}^{-1}$ will converge in Rat $_{d^{m-n}}$. But properness of the iteration map $\mathrm{Rat}_{d} \rightarrow \operatorname{Rat}_{d^{m-n}}$ [4, Corollary 0.3] implies that the sequence $A_{n, k} \circ f_{k} \circ A_{n, k}^{-1}$ must also converge uniformly to some rational function $g \in$ Rat $_{d}$. The continuity of measures within $\operatorname{Rat}_{d}$ then implies that $\mu=\lim _{k \rightarrow \infty}\left(A_{n, k}^{-1}\right)_{*} \mu_{g}$. The sequence $\left\{A_{n, k}\right\}$ must diverge in $\operatorname{Rat}_{1}$ (because the sequence $\left\{f_{k}\right\}$ diverges in $\mathrm{Rat}_{d}$ ), so the limiting measure $\mu$ will be concentrated at a single point.

It remains to treat the case where

$$
A_{m, k} \circ A_{n, k}^{-1}
$$

diverges in Rat for all $m>n \geqslant n_{0}$. A diagonalization argument allows us to assume that the limit exists in $\overline{\mathrm{Rat}}_{1}$, and we set

$$
a_{m, n}:=\lim _{k \rightarrow \infty} A_{m, k} \circ A_{n, k}^{-1}(p)
$$

for all but one point $p$ in $\mathbb{P}^{1}$, say $p=h_{m, n}$. Recall that we continue to assume that $\operatorname{deg} \phi_{n} \neq o\left(d^{n}\right)$ as $n \rightarrow \infty$, so there is a constant $0<\kappa<1$ such that $\operatorname{deg} \phi_{n}=\kappa d^{n}$ for all $n \geqslant n_{0}$. We wish to show that $\mu=\lim \mu_{k}$ is purely atomic. For the sake of a contradiction, we suppose otherwise and write

$$
\mu=v+\tilde{v},
$$

where $\tilde{v}$ is a countable sum of atoms and $v=\mu-\tilde{v}$ is a nonzero positive measure with no atoms. Similarly, write $\mu_{E_{n}}=v^{n}+\tilde{v}^{n}$, where $v^{n}$ and $\tilde{v}^{n}$ are the 'diffuse 
part' and the 'atomic part' of $\mu_{E_{n}}$, respectively. Applying Theorem 2.4 to the $n$th iterates $f_{k}^{n}$ and comparing diffuse parts, we find that

$$
v=\frac{1}{d^{n}} \phi_{n}^{*} \nu^{n} \Rightarrow 0<v\left(\mathbb{P}^{1}\right)=\frac{\operatorname{deg} \phi_{n}}{d^{n}} \nu^{n}\left(\mathbb{P}^{1}\right)=\kappa \cdot v^{n}\left(\mathbb{P}^{1}\right)
$$

for all $n \geqslant n_{0}$. Hence, there exists $N$ such that

$$
\sum_{n=n_{0}}^{N} v^{n}\left(\mathbb{P}^{1}\right) \geqslant 2
$$

Fix a small $\varepsilon>0$. For each pair $n_{0} \leqslant m, n \leqslant N$ with $m \neq n$, choose small pairwise disjoint closed disks $D_{m, n}$ and $D_{m, n}^{\prime}$ around $a_{m, n}$ and $h_{m, n}$, respectively. Let $U$ be the complement of all of these disks in $\mathbb{P}^{1}$. Since $v^{n}$ is atomless, by shrinking $D_{m, n}$ and $D_{m, n}^{\prime}$ as needed we may assume that

$$
v^{n}(U)>v^{n}\left(\mathbb{P}^{1}\right)-\frac{\varepsilon}{2^{n}} \quad\left(n_{0} \leqslant n \leqslant N\right) .
$$

Weak convergence of measures $\left(A_{n, k}\right)_{*} \mu_{k} \rightarrow \mu_{E_{n}}=v^{n}+\tilde{v}^{n}$ implies that

$$
\left(A_{n, k}\right)_{*} \mu_{k}(U)>v^{n}\left(\mathbb{P}^{1}\right)-\frac{\varepsilon}{2^{n}}
$$

for all sufficiently large $k$ and all $n_{0} \leqslant n \leqslant N$. (Restricting to finitely many $n$ allows us to do this uniformly.)

For distinct indices $n_{0} \leqslant m, n \leqslant N$, we have constructed $U$ to be disjoint from $D_{m, n}^{\prime}$. It follows that $A_{m, k} \circ A_{n, k}^{-1}(U) \subset D_{m, n}$ for all $k \gg 0$, and hence $U \cap\left(A_{m, k} \circ\right.$ $\left.A_{n, k}^{-1}(U)\right)=\varnothing$ for all sufficiently large $k$. Therefore, the sets

$$
A_{n_{0}, k}^{-1}(U), A_{n_{0}+1, k}^{-1}(U), \ldots, A_{N, k}^{-1}(U)
$$

are pairwise disjoint for all $k \gg 0$. (Again, restricting to finitely many sets allows us to do this uniformly.) But then

$$
\mu_{k}\left(\mathbb{P}^{1}\right) \geqslant \sum_{n=n_{0}}^{N} \mu_{k}\left(A_{n, k}^{-1}(U)\right)>\sum_{n=n_{0}}^{N}\left(v^{n}\left(\mathbb{P}^{1}\right)-\varepsilon / 2^{n}\right)>2-\varepsilon>1,
$$

contradicting the fact that $\mu_{k}$ is a probability measure. This completes the proof of Theorem A.

REMARK 2.6. In the case where the sequence $f_{k}$ lies in a meromorphic family $f_{t}$, the condition that $\operatorname{deg} \phi_{n} \neq o\left(d^{n}\right)$ is characterized in the proof of Proposition 4.7(2), in terms of dynamics on the Berkovich $\mathbf{P}^{1}$. 


\section{One-parameter families and complex surfaces}

In this section, we carry out Step 1 in the proof of Theorem B. To start, we consider a meromorphic family $\left\{f_{t}: t \in \mathbb{D}\right\}$ of rational functions of degree $d \geqslant 2$ and set up a geometric framework in which to talk about pullback of measures when $t=0$. Under the hypothesis of Theorem B, the family $f_{t}$ defines a holomorphic disk in $\overline{\mathrm{Rat}}_{d}$ with $f_{0} \in \partial \mathrm{Rat}_{d}$. It is convenient to package the given one-parameter family into one map on the complex surface $X=\mathbb{D} \times \mathbb{P}^{1}$, as

$$
F: X \rightarrow X,
$$

defined by $F(t, x)=\left(t, f_{t}(x)\right)$ for $t \neq 0$. The map $F$ extends to a meromorphic map on the surface $X$ with a finite set of indeterminacy points in the central fiber $X_{0}:=\{0\} \times \mathbb{P}^{1}$. The indeterminacy points coincide with roots of the polynomial gcd $H_{f_{0}}$ defined in Section 2.1. On any compact subset of $\mathbb{P}^{1} \backslash\left\{H_{f_{0}}=0\right\}$, the functions $f_{t}$ converge uniformly to the reduction $\phi_{f_{0}}$ as $t \rightarrow 0$.

3.1. The modified surface $Y$. We now construct the surface $Y$ that was alluded to in the introduction.

Proposition 3.1. There is a minimal complex modification $\pi: Y \rightarrow X$ such that the induced rational map

$$
F: X \rightarrow Y
$$

is nonconstant on the central fiber $X_{0}$. The minimal modification is unique up to isomorphism of fibered surfaces, possibly after shrinking the base $\mathbb{D}$. If $\phi_{f_{0}}$ is nonconstant, then $Y=X$. Otherwise, the central fiber of the surface $Y$ is reduced and has exactly two irreducible components.

REMARK 3.2. The surface $Y$ may be singular. We give a necessary and sufficient condition for nonsingularity in the course of the proof of the proposition.

EXAMPLE 3.3. Take $f_{t}(z)=t z^{2}$ for $t$ in the unit disk, and let $A_{t}(z)=t \frac{1}{z}$, so $A_{t} \circ$ $f_{t}$ has nonconstant reduction. The rational map $F: X \rightarrow X$ collapses the central fiber $X_{0}$ to the origin of $X_{0}$. The minimal modification $Y$ has local coordinates $(t, w)$ near the exceptional curve, where $w=t \frac{1}{z}$. Equivalently, $t=z w$, which is the standard equation for a blow-up at the origin of the surface $X=\mathbb{D} \times \mathbb{P}^{1}$ with coordinates $(t, z)$.

Proof of the Proposition. Evidently the surface $Y=X$ satisfies all of the desired properties if $\phi_{f_{0}}$ is nonconstant. 
For the remainder of the proof, we assume that $\phi_{f_{0}}$ is constant. Lemma 2.1 asserts that there is a Möbius transformation $A_{t}(z)$ with meromorphic coefficients such that $A_{t} \circ f_{t}$ converges to a point of $\overline{\mathrm{Rat}}_{1}$ with nonconstant reduction. If we take $(t, z)$ to be coordinates on $X=\mathbb{D} \times \mathbb{P}^{1}$ near a point of the central fiber $X_{0}$, then we may construct the surface $Y$ locally as a subvariety of $\left(\mathbb{D} \times \mathbb{P}^{1}\right) \times \mathbb{P}^{1}$ using the equation $A_{t}(z)=w$, where $w$ is an affine coordinate for the final $\mathbb{P}^{1}$ factor. Define $\pi: Y \rightarrow X$ to be projection on the $\left(\mathbb{D} \times \mathbb{P}^{1}\right)$ factor of the ambient space.

Note that $A_{t}$ is invertible for $t$ sufficiently small and nonzero, so $\pi$ is an isomorphism away from the central fibers of $X$ and $Y$. As $f_{t}$ has constant reduction, $A_{t}$ must have constant reduction too. Without loss of generality, we can assume that $A_{t} \rightarrow 0$ as $t \rightarrow 0$ away from finitely many points of $\mathbb{P}^{1}$. Thus we can write

$$
A_{t}(z)=t^{n} \cdot \frac{\alpha z+\beta}{\gamma z+\delta}
$$

where $\alpha, \beta, \gamma, \delta$ are meromorphic functions of $t$, holomorphic at $t=0$, and they satisfy $\alpha \delta-\beta \gamma \nrightarrow 0$ as $t \rightarrow 0$. Thus, a local equation for the surface $Y$ is

$$
(\gamma z+\delta) w=t^{n}(\alpha z+\beta) .
$$

Setting $t=0$ exhibits the two rational components of the central fiber of $Y$-one parameterized by $z$ and the other by $w$-and also shows that the central fiber is reduced. (Moreover, $Y$ is nonsingular if and only if $n=1$.)

We now prove uniqueness and minimality of this modification, simultaneously. Suppose that $\pi^{\prime}: Y^{\prime} \rightarrow X$ is a minimal modification of $X$ such that the induced rational map $F: X \rightarrow Y^{\prime}$ is nonconstant. Then $Y^{\prime}$ must have exactly two distinct components in its central fiber: the proper transform of the central fiber of $X$ and the image of the central fiber of $X$ under $F$. For if there were another component, we could blow it down and violate minimality. Working in coordinates at a smooth point of the exceptional divisor of $Y^{\prime}$, we observe that $F(t, z)=\left(t, g_{t}(z)\right)$ for some meromorphic family of rational functions $g_{t}$. For $t$ away from 0 , the function $g_{t}$ must agree with $f_{t}$ up to a coordinate change on the target, so there is a meromorphic family of Möbius transformations $B_{t}$ such that $B_{t} \circ f_{t}=g_{t}$. That is, the surface $Y^{\prime}$ is constructed locally via the equation $w^{\prime}=B_{t}(z)$ as in the previous paragraphs. Since $g_{t}$ has nonconstant reduction, Lemma 2.1 implies that $A_{t} \circ B_{t}^{-1}$ converges in $\mathrm{Rat}_{1}$ as $t \rightarrow 0$. In particular, this means that $Y^{\prime}$ and $Y$ are isomorphic via the fibered isomorphism $A_{t} \circ B_{t}^{-1}$. We conclude that $\pi: Y \rightarrow X$ is minimal and unique up to fibered isomorphism.

For the remainder of the paper, we fix the following notation. We choose a family $A_{t}$ of Möbius transformations such that $A_{t} \circ f_{t}$ converges to a point $\Phi \in \overline{\mathrm{Rat}}_{d}$ with gcd $H$ and reduction $\phi$ of degree $>0$. If the reduction of $f_{0}$ 


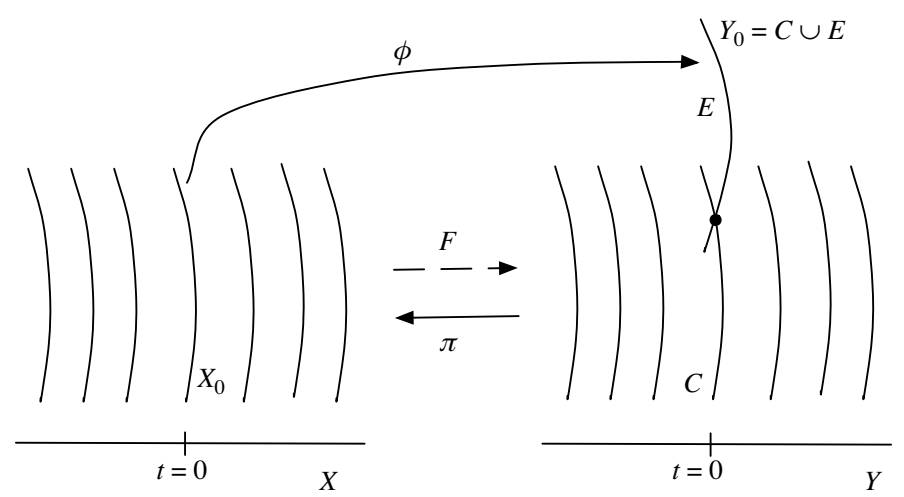

Figure 1: The surface map $F: X \rightarrow Y$ when the given reduction $\phi_{f_{0}}$ is constant.

is nonconstant, we let $A_{t}$ be the identity for all $t$. This choice gives rise to a minimal modification $\pi: Y \rightarrow X$ as in the above proof. If the central fiber $Y_{0}$ has two components, we view $Y$ as the blow-up of $X$ along an ideal supported at a single point in the central fiber of $X_{0}$. Let $E$ denote the exceptional curve of the projection $\pi: Y \rightarrow X$ and let $C$ be the other component of $Y_{0}$ (identified with the original fiber $X_{0}$ ); see Figure 1 . There are two natural projections from $Y_{0}$ to its irreducible components: the modification $\pi$ induces a projection $\pi: Y_{0} \rightarrow C$ collapsing $E$ to a point, and $\pi_{E}: Y_{0} \rightarrow E$, which is the unique continuous projection that is the identity on $E$.

3.2. Weak limits of measures on $\boldsymbol{Y}$. Suppose that $\mu_{t}$ is a family of probability measures on the fibers $Y_{t}$ on the surface $Y$. We say $\mu_{0}$ on $Y_{0}$ is a weak limit of the measures $\mu_{t}$ if there is a sequence $t_{n} \rightarrow 0$ such that

$$
\int_{Y_{t_{n}}} \psi \mu_{t_{n}} \rightarrow \int_{Y_{0}} \psi \mu_{0}
$$

for every continuous function $\psi$ on $Y$. If $Y=X=\mathbb{D} \times \mathbb{P}^{1}$, this notion of weak limit agrees with the usual notion for measures on a single $\mathbb{P}^{1}$. If $Y \neq X$, this notion of convergence coincides with $\left\{A_{t_{n}}\right\}$-weak convergence to the paired measure $\left(\pi_{*} \mu_{0},\left(\pi_{E}\right)_{*} \mu_{0}\right)$ on $C \cup E=Y_{0}$, as the following lemma shows; recall the definitions from Section 2.4.

Lemma 3.4. Suppose that $Y \neq X$, and so $Y_{0}=C \cup E$. A measure $\mu_{0}$ on $Y_{0}$ is a weak limit of measures $\mu_{t}$ on $Y_{t}$ if and only if there is a sequence $t_{n}$ of parameters such that $\mu_{t_{n}}$ converges $\left\{A_{t_{n}}\right\}$-weakly to the paired measure $\left(\pi_{*} \mu_{0},\left(\pi_{E}\right)_{*} \mu_{0}\right)$ on $C, E$. 
Proof. Assume first that $\mu_{0}$ is the weak limit of measures $\mu_{t_{n}}$ on fibers $Y_{t_{n}}$. We identify the curves $C$ and $E$ with abstract copies of $\mathbb{P}^{1}$, with coordinates $z \in C$ and $w \in E$. Let $\psi_{C}$ be any continuous function on $C$, and let $\psi_{E}$ be any continuous function on $E$. We define continuous functions on $Y$ by

$$
\tilde{\psi}_{C}(y)=\psi_{C}(z)
$$

when $\pi(y)=(t, z) \in X=\mathbb{D} \times \mathbb{P}^{1}$, and

$$
\tilde{\psi}_{E}(y)= \begin{cases}\psi_{E}(w) & \text { if } \pi(y)=\left(t, A_{t}^{-1}(w)\right), t \neq 0, \text { on } X=\mathbb{D} \times \mathbb{P}^{1} \\ \psi_{E}(w) & \text { for } w \in E \\ \psi_{E}(p) & \text { on } C\end{cases}
$$

where $p \in Y_{0}$ denotes the point of intersection of $C$ and $E$.

Then

$$
\begin{aligned}
\int_{C} \psi_{C} \mu_{t_{n}} & =\int_{Y_{t_{n}}} \tilde{\psi}_{C} \mu_{t_{n}} \\
& \longrightarrow \int_{Y_{0}} \tilde{\psi}_{C} \mu_{0} \\
& =\int_{C \backslash\{p\}} \tilde{\psi}_{C} \mu_{0}+\tilde{\psi}_{C}(p) \mu_{0}(\{p\})+\int_{E \backslash\{p\}} \tilde{\psi}_{C} \mu_{0} \\
& =\int_{C \backslash\{p\}} \psi_{C} \mu_{0}+\psi_{C}(p) \mu_{0}(E)=\int_{C} \psi_{C}\left(\pi_{*} \mu_{0}\right),
\end{aligned}
$$

demonstrating the weak convergence of $\mu_{t_{n}} \rightarrow \pi_{*} \mu_{0}$.

Also, we have

$$
\begin{aligned}
\int_{E} \psi_{E}(w) A_{t_{n} *} \mu_{t_{n}}(w) & =\int_{Y_{t_{n}}} \tilde{\psi}_{E}\left(t_{n}, A_{t_{n}}^{-1}(w)\right) A_{t_{n} *} \mu_{t_{n}}(w) \\
& =\int_{Y_{t_{n}}} \tilde{\psi}_{E}\left(t_{n}, z\right) \mu_{t_{n}}(z) \\
& \longrightarrow \int_{Y_{0}} \tilde{\psi}_{E} \mu_{0} \\
& =\int_{E \backslash\{p\}} \tilde{\psi}_{E} \mu_{0}+\tilde{\psi}_{E}(p) \mu_{0}(\{p\})+\int_{C \backslash\{p\}} \tilde{\psi}_{E} \mu_{0} \\
& =\int_{E \backslash\{p\}} \psi_{E} \mu_{0}+\psi_{E}(p) \mu_{0}(C)=\int_{E} \psi_{E}\left(\pi_{E *} \mu_{0}\right),
\end{aligned}
$$

demonstrating the weak convergence of $A_{t_{n} *} \mu_{t_{n}} \rightarrow \pi_{E *} \mu_{0}$. 
For the reverse implication, assume that $\mu_{0}$ is a probability measure on $Y_{0}$ such that $\left\{\mu_{t_{n}}\right\}$ converges $\left\{A_{t_{n}}\right\}$-weakly to the paired measure $\left(\pi_{*} \mu_{0},\left(\pi_{E}\right)_{*} \mu_{0}\right)$. Let $\psi$ be any continuous function on $Y$ and fix $\varepsilon>0$. With a partition of unity, we may write $\psi=\psi_{C}+\psi_{E}+\psi_{p}$, where $\psi_{C} \equiv 0$ on $E, \psi_{E} \equiv 0$ on $C$, and $\psi_{p}$ is supported on a small neighborhood of $\{p\}=C \cap E$. We choose the neighborhood of $p$ small enough that

$$
\left|\int_{Y_{0}} \psi_{p} \mu_{0}-\psi(p) \mu_{0}(\{p\})\right|<\varepsilon .
$$

By uniform continuity of $\psi_{C}$ near the central fiber $Y_{0}$, the weak convergence of $\mu_{t_{n}}$ to $\pi_{*} \mu_{0}$ guarantees that

$$
\left|\int_{Y_{t_{n}}} \psi_{C} \mu_{t_{n}}-\int_{C} \psi_{C}\left(\pi_{*} \mu_{0}\right)\right|<\varepsilon
$$

for all $n$ sufficiently large. Note that

$$
\int_{C} \psi_{C}\left(\pi_{*} \mu_{0}\right)=\int_{Y_{0}} \psi_{C} \mu_{0}
$$

because $\psi_{C} \equiv 0$ on $E$. Now let $\phi_{E}=\psi_{E} \mid E$. By continuity of $\psi_{E}$, with coordinate $w=A_{t}(z)$ near $E$, we see that

$$
\left|\psi_{E}(t, z)-\phi_{E}\left(A_{t}(z)\right)\right|<\varepsilon
$$

on $Y_{t}$, uniformly for all $t$ small. Then the convergence of $A_{t_{n} *} \mu_{t_{n}}$ to $\pi_{E *} \mu_{0}$ implies that

$$
\left|\int_{Y_{t_{n}}} \psi_{E} \mu_{t_{n}}-\int \phi_{E}\left(\pi_{E *} \mu_{0}\right)\right|<2 \varepsilon
$$

for all $n$ sufficiently large. Note that

$$
\int \phi_{E}\left(\pi_{E *} \mu_{0}\right)=\int_{Y_{0}} \psi_{E} \mu_{0}
$$

because $\psi_{E} \equiv 0$ on $C$. At the intersection point $\{p\}=C \cap E$, we have

$$
\mu_{0}(\{p\})=\pi_{*} \mu_{0}(\{p\})-\pi_{E *} \mu_{0}(E \backslash\{p\})=\pi_{E *} \mu_{0}(\{p\})-\pi_{*} \mu_{0}(C \backslash\{p\}) .
$$

It follows from weak convergence to the paired measure and (3.2.1) that

$$
\left|\int_{Y_{t_{n}}} \psi_{p} \mu_{t_{n}}-\int_{Y_{0}} \psi_{p} \mu_{0}\right|<2 \varepsilon
$$

for all $n$ large. Putting the pieces together, and shrinking the neighborhood of $p$, we conclude that

$$
\int_{Y_{t_{n}}} \psi \mu_{t_{n}}=\int_{Y_{t_{n}}}\left(\psi_{C}+\psi_{E}+\psi_{p}\right) \mu_{t_{n}} \longrightarrow \int_{Y_{0}} \psi \mu_{0} .
$$


3.3. Pullback of measures from $\boldsymbol{Y}_{\mathbf{0}}$ to $\boldsymbol{X}_{\mathbf{0}}$. For any Borel probability measure $\mu$ on the central fiber $Y_{0}$ of $Y$, we can define a measure $F^{*} \mu$ on the central fiber $X_{0}$ of $X$ of total mass $d$. We use the degenerate pullbacks defined in Section 2.3. If $Y=X$, we simply set

$$
F^{*} \mu:=\Phi^{*} \mu=\phi^{*} \mu+\sum_{x \in \mathbb{P}^{1}} s(x) \delta_{x} .
$$

For the case $Y \neq X$, recall that the projection $\pi_{E}: Y_{0} \rightarrow E$ collapses $C$ to a point. We define

$$
F^{*} \mu:=\Phi^{*}\left(\pi_{E *} \mu\right)=\phi^{*}\left(\pi_{E *} \mu\right)+\sum_{x \in \mathbb{P}^{1}} s(x) \delta_{x} .
$$

We already know that weak limits of maximal measures satisfy a pairedmeasure pullback formula (Theorem 2.4). Translating into our surface framework with Lemma 3.4, we immediately obtain the main result of this section:

THEOREM 3.5. Any weak limit $\mu_{0}$ of the maximal measures $\mu_{t}$ on the central fiber $Y_{0}$ of $Y$ will satisfy the pullback equation

$$
\frac{1}{d} F^{*} \mu_{0}=\pi_{*} \mu_{0}
$$

on the central fiber $X_{0}$ of $X$.

\section{Dynamics and $\boldsymbol{\Gamma}$-measures on the Berkovich projective line}

In this section, we quantize a dynamical system $f$ on the Berkovich projective line and describe the solutions to a system of pullback formulas, thereby completing Step 2 of our program outlined in the introduction. Throughout, we let $k$ be an algebraically closed field of characteristic zero that is complete with respect to a nontrivial non-Archimedean absolute value. Only the case where $k$ has residue characteristic zero is necessary for our application; however, with essentially no extra work, we obtain a more general result. The Berkovich projective line over $k$ will be denoted as $\mathbf{P}^{1}$ for brevity (as opposed to $\mathbf{P}_{k}^{1, \text { an }}$ ) .

4.1. Basic background. The Berkovich projective line is a complicated object from an analytic viewpoint; a careful development is given in [1]. However, the arguments in the next few subsections will typically only require basic topological properties of $\mathbf{P}^{1}$ and of its self-maps. For a self-contained and detailed summary with complete references, see $[\mathbf{8}$, Sections 2,3$]$. To aid the reader, we give our own intuitive summary now. 
The Berkovich projective line $\mathbf{P}^{1}$ over $k$ is a connected compact Hausdorff topological space with a 'tree structure': it is uniquely arcwise connected. It contains a canonical homeomorphic copy of the space $\mathbb{P}^{1}(k)$ with the metric topology induced from $k$; we refer to these as the classical or type I points of $\mathbf{P}^{1}$. These points all lie at the 'ends' of the tree. The branch points of $\mathbf{P}^{1}$ are called type II points; they correspond bijectively to closed disks in $k$ with radius in the value group $\left|k^{\times}\right|$. In particular, we will make use of the 'Gauss point' of $\mathbf{P}^{1}$ corresponding to the closed unit disk in $k$. For each type II point $\zeta$, the connected components of $\mathbf{P}^{1} \backslash\{\zeta\}$ are examples of 'open Berkovich disks'.

A rational function $f: \mathbb{P}_{k}^{1} \rightarrow \mathbb{P}_{k}^{1}$ extends uniquely to a morphism $f: \mathbf{P}^{1} \rightarrow \mathbf{P}^{1}$. If $f$ is nonconstant, there is a multiplicity (or local degree) function $m_{f}: \mathbf{P}^{1} \rightarrow\{1$, $\ldots, \operatorname{deg}(f)\}$ that extends the usual algebraic multiplicity for $f: \mathbb{P}_{k}^{1} \rightarrow \mathbb{P}_{k}^{1}$. As one expects, 'most' points $x \in \mathbf{P}^{1}$ have $m_{f}(x)=1$, and each point $y \in \mathbf{P}^{1}$ has exactly $\operatorname{deg}(f)$ preimages under $f$ when counted with multiplicities.

4.2. Vertex sets and measures. A vertex set for $\mathbf{P}^{1}$ is a finite nonempty set of type II points, which we denote by $\Gamma$. The connected components of $\mathbf{P}^{1} \backslash \Gamma$ will be referred to as $\Gamma$-domains. When a $\Gamma$-domain has only one boundary point, we call it a $\Gamma$-disk. Write $\mathcal{S}(\Gamma)$ for the partition of $\mathbf{P}^{1}$ consisting of the elements of $\Gamma$ and all of its $\Gamma$-domains.

Let $\left(\mathbf{P}^{1}, \Gamma\right)$ be the measurable space structure on $\mathbf{P}^{1}$ equipped with the $\sigma$ algebra generated by the power set of $\mathcal{S}(\Gamma)$. A measurable function on $\left(\mathbf{P}^{1}, \Gamma\right)$ will be called $\Gamma$-measurable. The space of complex measures on $\left(\mathbf{P}^{1}, \Gamma\right)$ will be denoted as $M(\Gamma)$, and we call any such measure a $\Gamma$-measure. We write $M^{\ell}(\Gamma)$ for the convex subspace of $M(\Gamma)$ consisting of positive measures of volume $\ell$.

ReMARK 4.1. A function $\phi: \mathbf{P}^{1} \rightarrow \mathbb{C}$ is $\Gamma$-measurable if and only if it is constant on elements of $\mathcal{S}(\Gamma)$.

Suppose that $\Gamma \subset \Gamma^{\prime}$ are two vertex sets. If we write $\pi: \mathbf{P}^{1} \rightarrow \mathbf{P}^{1}$ for the identity morphism, then $\pi:\left(\mathbf{P}^{1}, \Gamma^{\prime}\right) \rightarrow\left(\mathbf{P}^{1}, \Gamma\right)$ is a measurable morphism. In particular, the projection

$$
\pi_{*}: M\left(\Gamma^{\prime}\right) \rightarrow M(\Gamma)
$$

is $\mathbb{C}$-linear and preserves positivity and volume of measures.

4.3. Pulling back measures by a rational function. Throughout this section we assume that $f: \mathbf{P}^{1} \rightarrow \mathbf{P}^{1}$ is a rational function of degree $d \geqslant 2$. Suppose that $\Gamma=\{\zeta\}$ is a singleton vertex set, and let $\Gamma^{\prime}=\{\zeta, f(\zeta)\}$ be a second vertex set. 
For the applications in this article, we will only need to consider vertex sets of cardinality one or two.

Now we define a pullback map $f^{*}: M\left(\Gamma^{\prime}\right) \rightarrow M(\Gamma)$. As a first step, we define certain multiplicities $m_{U, V} \in\{0,1, \ldots, d\}$ for each $U \in \mathcal{S}\left(\Gamma^{\prime}\right)$ and $V \in \mathcal{S}(\Gamma)$. If $V=\{\zeta\}$, set $m_{U, V}=m_{f}(\zeta)$, the usual local degree of $f$ at $\zeta$. For a $\Gamma$-disk $V$, we may write $V=D(\vec{v})$ for some tangent vector $\vec{v} \in T \mathbf{P}_{\zeta}^{1}$. Set $\bar{f}(V)=$ $D(T f(\vec{v}))$. Write $m_{f}(V)$ and $s_{f}(V)$ for the directional and surplus multiplicities for $f$ associated with $V$. (See [8, Section 3].) By definition, we have

$$
\#\left(f^{-1}(y) \cap V\right)= \begin{cases}m_{f}(V)+s_{f}(V) & \text { if } y \in \bar{f}(V) \\ s_{f}(V) & \text { if } y \notin \bar{f}(V) .\end{cases}
$$

Here we count each preimage $x$ with multiplicity $m_{f}(x)$. Since $\bar{f}(V)$ is a union of elements of $\mathcal{S}\left(\Gamma^{\prime}\right)$, the function $y \mapsto \#\left(f^{-1}(y) \cap V\right)$ is constant on elements of $\mathcal{S}\left(\Gamma^{\prime}\right)$. For each $U \in \mathcal{S}\left(\Gamma^{\prime}\right)$, define $m_{U, V}$ to be this constant value. The following lemma gives a compatibility relation among the multiplicities $m_{U, V}$.

Lemma 4.2. For each $U \in \mathcal{S}\left(\Gamma^{\prime}\right)$, we have

$$
\sum_{V \in \mathcal{S}(\Gamma)} m_{U, V}=\operatorname{deg}(f)
$$

Proof. Choose a point $y \in U$. For each $V \in \mathcal{S}(\Gamma)$, we have that $m_{U, V}=$ $\#\left(f^{-1}(y) \cap V\right)$. Since $f$ is everywhere $\operatorname{deg}(f)$-to-one, the result follows.

REMARK 4.3. The notation $m_{U, V}$ is backward from the point of view of mappings: it is the local degree of the map $\left(f^{-1}(U) \cap V\right) \stackrel{f}{\rightarrow} U$. However, in the sequel [6], we interpret this quantity in terms of a transition probability for passing from 'state $U$ ' to 'state $V$ ' in a certain random process. Specifically, the fraction $p_{U, V}:=m_{U, V} / d$ is the probability that a randomly chosen preimage of a point $y \in U$ lies in $V$. The preceding lemma shows that these probabilities sum to 1 when $U$ is fixed and $V$ varies.

For a measurable function $\phi:\left(\mathbf{P}^{1}, \Gamma\right) \rightarrow \mathbb{C}$, we define a $\Gamma^{\prime}$-measurable function $f_{*} \phi$ by

$$
f_{*} \phi(U)=\sum_{W \in \mathcal{S}(\Gamma)} m_{U, W} \cdot \phi(W) \quad\left(U \in \mathcal{S}\left(\Gamma^{\prime}\right)\right) .
$$

Here we have abused notation by writing $f_{*} \phi(U)$ for the constant value of $f_{*} \phi$ on $U$, and similarly for $\phi(W)$. Note that the sum defining $f_{*} \phi(U)$ is finite by Lemma 4.2. 
If $\phi$ is a bounded $\Gamma$-measurable function, then $\left\|f_{*} \phi\right\| \leqslant d\|\phi\|$, where we have written $\|\cdot\|$ for the sup norm. For each $v \in M\left(\Gamma^{\prime}\right)$, the linear functional $\phi \mapsto \int f_{*} \phi v$ is bounded, and by duality there exists a $\Gamma$-measure $f^{*} v$ satisfying $\int \phi f^{*} v=\int f_{*} \phi v$ for all bounded $\Gamma$-measurable functions $\phi$. Evidently $f^{*}$ : $M\left(\Gamma^{\prime}\right) \rightarrow M(\Gamma)$ preserves positivity of measures, and Lemma 4.2 shows that $f^{*}$ carries $M^{\ell}\left(\Gamma^{\prime}\right)$ into $M^{\ell d}(\Gamma)$ for each $\ell \in \mathbb{C}$. In particular, $\frac{1}{d} f^{*}$ maps probability measures to probability measures.

4.4. The equilibrium and exceptional $\boldsymbol{\Gamma}$-measures. For a given rational function $f: \mathbf{P}^{1} \rightarrow \mathbf{P}^{1}$ of degree $d \geqslant 2$ and finite vertex set $\Gamma$, there are two distinguished $\Gamma$-measures that will play a key role in our theory.

Write $\mu_{f}$ for the equilibrium measure on $\mathbf{P}^{1}$ relative to $f$ [11]. (Another common name in the literature is 'canonical measure' [1, Section 10].) It is the unique Borel probability measure $v$ that satisfies $f^{*} v=d \cdot v$ and that does not charge classical points of $\mathbf{P}^{1}$ [11, Thm. A]. Here $f^{*}$ is the usual pullback operator for Borel measures on $\mathbf{P}^{1}$-not the one defined in Section 4.3. For a vertex set $\Gamma$, we define the equilibrium $\Gamma$-measure $\omega_{f, \Gamma}$ by the formula

$$
\omega_{f, \Gamma}(U):=\mu_{f}(U)
$$

for each $U \in \mathcal{S}(\Gamma)$.

By construction, the equilibrium measure $\omega_{f, \Gamma}$ must be supported on a countable subset of $\mathcal{S}(\Gamma)$. Also, the measure $\omega_{f, \Gamma}$ has total mass 1 . Indeed, the Julia set of $f$ on $\mathbf{P}^{1}$ is precisely the support of the measure $\mu_{f}$ [1, Section 10.5]. For each $\Gamma$-domain $U$ that intersects the Julia set, there is an iterate $f^{n}$ that maps $U$ over $\Gamma$. So only countably many $\Gamma$-domains can intersect the Julia set. In other words, there cannot be a collection $X$ of $\Gamma$-domains which has positive measure for $\omega_{f, \Gamma}$ but with $\omega_{f, \Gamma}(U)=0$ for all $U \in X$. (Note that $\omega_{f, \Gamma}(\Gamma)>0$ if and only if the Julia set of $f$ lies in $\Gamma[1,11]$.)

LeMma 4.4. Let $f: \mathbf{P}^{1} \rightarrow \mathbf{P}^{1}$ be a rational function of degree $d \geqslant 2$, let $\Gamma=\{\zeta\}$ be a singleton vertex set, let $\Gamma^{\prime}=\{\zeta, f(\zeta)\}$, and let $\pi_{*}$ and $f^{*}$ be the operators defined in the previous section. Then $\pi_{*} \omega_{f, \Gamma^{\prime}}=\omega_{f, \Gamma}$ and $f^{*} \omega_{f, \Gamma^{\prime}}=d \cdot \pi_{*} \omega_{f, \Gamma^{\prime}}$.

Proof. The statement about $\pi_{*}$ is immediate from the definitions.

Let $\phi: \mathbf{P}^{1} \rightarrow \mathbb{C}$ be a $\Gamma$-measurable function. It is also Borel measurable on $\mathbf{P}^{1}$ since each element of $\mathcal{S}(\Gamma)$ is either an open set or a point. The definitions of the multiplicities $m_{U, V}$ show that

$$
f_{*} \phi(y)=\sum_{f(x)=y} m_{f}(x) \phi(x) \quad\left(y \in \mathbf{P}^{1}\right),
$$


which agrees with the formula for the pushforward of Borel measurable functions. Since $f^{*} \mu_{f}=d \cdot \mu_{f}$ as Borel measures on $\mathbf{P}^{1}$, we find that

$$
\begin{aligned}
\int \phi f^{*} \omega_{f, \Gamma^{\prime}}=\int f_{*} \phi \omega_{f, \Gamma^{\prime}} & =\int f_{*} \phi \mu_{f} \\
& =\int \phi f^{*} \mu_{f}=d \int \phi \mu_{f}=d \int \phi \pi_{*} \omega_{f, \Gamma^{\prime}}
\end{aligned}
$$

Hence $f^{*} \omega_{f, \Gamma^{\prime}}=d \cdot \pi_{*} \omega_{f, \Gamma^{\prime}}$ as elements of $M(\Gamma)$.

Suppose now that the rational function $f: \mathbf{P}^{1} \rightarrow \mathbf{P}^{1}$ has an exceptional orbit $\mathcal{E}$. The exceptional $\Gamma$-measure associated with the orbit $\mathcal{E}$ is defined to be the probability measure $\delta_{\mathcal{E}} \in M(\Gamma)$ given by

$$
\delta_{\mathcal{E}}(U)=\frac{\#(\mathcal{E} \cap U)}{\# \mathcal{E}} .
$$

REMARK 4.5. Recall that an exceptional orbit $\mathcal{E}$ is finite and $f^{-1}(\mathcal{E})=\mathcal{E}$. Since $k$ has characteristic zero, the function $f$ admits at most two classical exceptional points and at most one exceptional point in $\mathbf{P}^{1} \backslash \mathbb{P}^{1}(k)$ (necessarily of type II).

Lemma 4.6. Let $f: \mathbf{P}^{1} \rightarrow \mathbf{P}^{1}$ be a rational function of degree $d \geqslant 2$, let $\Gamma=\{\zeta\}$ be a singleton vertex set, and let $\Gamma^{\prime}=\{\zeta, f(\zeta)\}$. Suppose that $\mathcal{E}$ is an exceptional orbit for $f$. Write $\delta_{\mathcal{E}}$ and $\delta_{\mathcal{E}}^{\prime}$ for the associated probability measures with respect to $\Gamma$ and $\Gamma^{\prime}$, respectively. Then $\pi_{*} \delta_{\mathcal{E}}^{\prime}=\delta_{\mathcal{E}}$ and $f^{*} \delta_{\mathcal{E}}^{\prime}=d \cdot \pi_{*} \delta_{\mathcal{E}}^{\prime}$.

Proof. Since exceptional measures count the number of exceptional points, we evidently have $\pi_{*} \delta_{\mathcal{E}}^{\prime}=\delta_{\mathcal{E}}$. For the other equality, let $U \in \mathcal{S}(\Gamma)$. Then

$$
f^{*} \delta_{\mathcal{E}}^{\prime}(U)=\sum_{\substack{V \in \mathcal{S}\left(\Gamma^{\prime}\right) \\ V \subset f(U)}} m_{V, U} \frac{\#(\mathcal{E} \cap V)}{\# \mathcal{E}} .
$$

The quantity $m_{V, U}$ is the constant value of \# $\left(f^{-1}(y) \cap U\right)$ for $y \in V$, counted with multiplicities. In particular, if $c \in \mathcal{E} \cap V$, then $m_{V, U}=0$ or $d$, depending on whether $f^{-1}(c) \cap U$ is empty or not. Note also that $\#(\mathcal{E} \cap U)=\#(\mathcal{E} \cap f(U))$, since $\mathcal{E}$ is a totally invariant set. Hence,

$$
f^{*} \delta_{\mathcal{E}}^{\prime}(U)=\sum_{\substack{V \in \mathcal{S}\left(\Gamma^{\prime}\right) \\ V \subset f(U)}} d \frac{\#(\mathcal{E} \cap V)}{\# \mathcal{E}}=d \frac{\#(\mathcal{E} \cap U)}{\# \mathcal{E}}=d \cdot \pi_{*} \delta_{\mathcal{E}}^{\prime}(U) .
$$


4.5. Surplus equidistribution and surplus estimates. We now give two technical results that will be used to prove the main result in the next section. The first is of interest in its own right: it describes how surplus multiplicities of disks behave under iteration. The second gives a lower bound for the mass of a $\Gamma$-disk in terms of its surplus multiplicity.

Proposition 4.7 (Surplus Equidistribution). Let $f: \mathbf{P}^{1} \rightarrow \mathbf{P}^{1}$ be a rational function of degree $d \geqslant 2$ with associated equilibrium measure $\mu_{f}$. Let $U$ be an open Berkovich disk with boundary point $\zeta$. Suppose that the Julia set of $f$ is not equal to $\{\zeta\}$. Then exactly one of the following is true:

(1) The iterated surplus multiplicities of $U$ satisfy

$$
s_{f^{n}}(U)=\mu_{f}(U) \cdot d^{n}+o\left(d^{n}\right) .
$$

(2) The orbit $\mathcal{O}_{f}(\zeta)$ converges along the locus of total ramification to a classical exceptional orbit (of length 1 or 2), and

$$
s_{f^{n}}(U)=0 \text { and } \mu_{f}\left(f^{n}(U)\right)=1 \text { for all } n \geqslant 1 .
$$

Proof. The two cases of the proposition are mutually exclusive. For if (2) holds, then $s_{f}(U)=0$, so $f(U) \neq \mathbf{P}^{1}$. The relation $\mu_{f}=\frac{1}{d} f^{*} \mu_{f}$ of Borel measures yields

$$
\mu_{f}(U)=\frac{m_{f}(U)}{d} \mu_{f}(f(U))=\frac{m_{f}(U)}{d}>0 .
$$

But then (1) is contradicted.

In the remainder of the proof, let us assume that case (1) of the proposition does not hold. The equilibrium measure $\mu_{f}$ does not charge $\zeta$ by hypothesis on the Julia set of $f$. Let $y$ be an arbitrary point of $\mathbf{P}^{1}$ that is not a classical exceptional point. Using equidistribution of iterated preimages [11, Thm. A], we find that

$$
\mu_{f}(U)=\lim _{n \rightarrow \infty} \frac{\#\left(f^{-n}(y) \cap U\right)}{d^{n}}=\lim _{n \rightarrow \infty} \frac{\varepsilon(y, n, U) \cdot m_{f^{n}}(U)+s_{f^{n}}(U)}{d^{n}},
$$

where $\varepsilon(y, n, U)=1$ if $y \in \overline{f^{n}}(U)$ and 0 otherwise. We conclude that $m_{f^{n}}(U) \neq$ $o\left(d^{n}\right)$; for otherwise, we are in case (1) of the proposition.

Let $\zeta_{0}=\zeta$ and set $\zeta_{n}=f\left(\zeta_{n-1}\right)$ for each $n \geqslant 1$. We can write $\vec{v}_{n} \in T \mathbf{P}_{\zeta_{n}}^{1}$ for the tangent vector such that $D\left(\vec{v}_{0}\right)=U$ and $T f^{n}\left(\vec{v}_{0}\right)=\vec{v}_{n}$. Then

$$
m_{f^{n}}(U)=\prod_{i=0}^{n-1} m_{f}\left(D\left(\vec{v}_{i}\right)\right) .
$$


Each factor in the product is an integer in the range $1, \ldots, d$. If infinitely many of the multiplicities $m_{f}\left(D\left(\vec{v}_{i}\right)\right)$ are strictly smaller than $d$, then $m_{f^{n}}(U)=o\left(d^{n}\right)$. Thus $m_{f}\left(D\left(\vec{v}_{n}\right)\right)=d$ for all $n \gg 0$. As multiplicities are upper semicontinuous, this shows that $m_{f}\left(\zeta_{n}\right)=d$ for all $n$ sufficiently large, so the orbit $\mathcal{O}_{f}(\zeta)$ eventually lies in the locus of total ramification $\mathcal{R}_{f}^{\text {tot }}$.

We now show that $\mathcal{O}_{f}(\zeta)$ converges to a classical exceptional orbit. Let $n_{0}$ be such that $\zeta_{n} \in \mathcal{R}_{f}^{\text {tot }}$ for all $n \geqslant n_{0}$. The locus of total ramification is connected [8, Thm. 8.2], and any pair of points in $\mathcal{R}_{f}^{\text {tot }}$ lie at finite hyperbolic distance to each other unless one is a classical critical point. So it suffices to prove that the hyperbolic distance $\rho_{\mathbf{H}}\left(\zeta_{n_{0}}, \zeta_{n}\right)$ grows without bound as $n \rightarrow \infty$. For ease of notation, let us assume that $n_{0}=0$. Since $\zeta_{n}, \zeta_{n+1} \in \mathcal{R}_{f}^{\text {tot }}$, the entire segment connecting them must lie in the locus of total ramification as well. Hence $f$ maps $\left[\zeta_{n}, \zeta_{n+1}\right]$ injectively onto $\left[\zeta_{n+1}, \zeta_{n+2}\right]$. Moreover, $\rho_{\mathbf{H}}\left(\zeta_{n+1}, \zeta_{n+2}\right)=d \cdot \rho_{\mathbf{H}}\left(\zeta_{n}, \zeta_{n+1}\right)$. By induction, we see that

$$
\rho_{\mathbf{H}}\left(\zeta_{n+\ell}, \zeta_{n+\ell+1}\right)=d^{\ell} \cdot \rho_{\mathbf{H}}\left(\zeta_{n}, \zeta_{n+1}\right), \quad \ell=0,1,2, \ldots,
$$

so the locus of total ramification has infinite diameter. The locus of total ramification has at most two classical points in it; hence, some classical totally ramified point $c$ is an accumulation point of $\mathcal{O}_{f}(\zeta)$. By (weak) continuity of $f$, we find that $f(c) \in \mathcal{R}_{f}^{\text {tot }}$. So $c$ is exceptional of period one or two. The orbit $\mathcal{O}_{f}(\zeta)$ must actually converge to the orbit of $c$ since the latter is attractive.

Since $f$ has a classical exceptional point, it is conjugate either to a polynomial or to $z \mapsto z^{-d}$. We treat the former case and leave the latter to the reader. Without loss of generality, we now assume that $f$ is a polynomial and that $f^{n}(\zeta)$ converges to $\infty$ along the locus of total ramification. As $k$ has characteristic zero, the ramification locus near $\infty$ is contained in a strong tubular neighborhood of finite radius around $\left(\zeta_{0, R}, \infty\right)$ for some $R>1[9, \mathrm{Thm}$. F]. Since hyperbolic distance is expanding on the ramification locus, we see that $f^{n}(\zeta)$ converges to infinity along the segment $\left(\zeta_{0, R}, \infty\right)$. In particular, since the Julia set of $f$ is bounded away from $\infty$, and since $f$ preserves the partial ordering of points in the tree $\mathbf{P}^{1}$ (relative to the maximal point $\infty$ ), we see that $\zeta$ must lie above the entire Julia set. That is, every segment from a Julia point to $\infty$ must pass through $\zeta$.

Recall that $\Gamma=\{\zeta\}$, and that we are looking at a distinguished $\Gamma$-disk $U$. The previous paragraph shows that either $U$ does not meet infinity or it does not meet the Julia set (or both). In particular, since the Julia set and the point at infinity are totally invariant for $f$, we have $f^{n}(U) \neq \mathbf{P}^{1}$, and so the surplus multiplicities satisfy $s_{f^{n}}(U)=0$ for all $n \geqslant 1$. In fact, $U$ must meet the Julia set; otherwise, $\mu_{f}(U)=0$, and we are in case (1) of the proposition. Observe that $\zeta \in f^{n}(U)$ for each $n \geqslant 1$, so the entire Julia set of $f$ is contained in $f^{n}(U)$. This shows that $\mu_{f}\left(f^{n}(U)\right)=1$, and we are in case (2) of the proposition as desired. 
LeMma 4.8 (Surplus Estimate). Let $f: \mathbf{P}^{1} \rightarrow \mathbf{P}^{1}$ be a rational function of degree $d \geqslant 2$, and let $\Gamma=\{\zeta\}$ be a singleton vertex set. Set $\Gamma^{\prime}=\{\zeta, f(\zeta)\}$. (Note that $\Gamma=\Gamma^{\prime}$ is allowed.) For any $\Gamma$-disk $U$ and any $\Gamma^{\prime}$-measure solution $v$ to the equation $f^{*} v=d \cdot \pi_{*} v$, we find that

$$
v(U) \geqslant \frac{s_{f}(U)}{d} .
$$

Proof. For ease of notation, let us write $m=m_{f}(U)$ and $s=s_{f}(U)$. We may explicitly compute the multiplicities appearing in the pullback operator to be

$$
m_{V, U}= \begin{cases}m+s & \text { if } V \subset \bar{f}(U) \\ s & \text { if } V \not \subset \bar{f}(U) .\end{cases}
$$

Then for $\chi_{U}$ the characteristic function on the $\Gamma$-disk $U$,

$$
\begin{aligned}
d \cdot \pi_{*} v(U)=f^{*} v(U) & =\int_{\bar{f}(U)} f_{*} \chi_{U} v+\int_{\mathbf{P}^{1} \backslash \bar{f}(U)} f_{*} \chi_{U} v \\
& =(m+s) \cdot v(\bar{f}(U))+s \cdot v\left(\mathbf{P}^{1} \backslash \bar{f}(U)\right) \\
& =s+m \cdot v(\bar{f}(U)) \geqslant s .
\end{aligned}
$$

Dividing by $d$ gives the result.

4.6. Simultaneous solutions to iterated pullback formulas. The equation $f^{*} v=d \cdot \pi_{*} v$ does not necessarily have a unique solution $v \in M^{1}(\Gamma)$ as one might expect by analogy with the standard setting. However, the solution does become essentially unique if we impose all pullback relations $\left(f^{n}\right)^{*} v=d^{n} \cdot \pi_{n *} v$ for $n=1,2,3, \ldots$

Let $\Gamma=\{\zeta\}$ be a singleton vertex set for $\mathbf{P}^{1}$. Let $\Gamma_{n}=\left\{\zeta, f^{n}(\zeta)\right\}$ for each $n \geqslant 1$, and write $\left(f^{n}\right)^{*}$ and $\pi_{n *}$ for the pullback and pushforward operators relative to $\Gamma$ and $\Gamma_{n}$, respectively. We define a set of $\Gamma$-measures $\Delta_{f} \subset M^{1}(\Gamma)$ by

$$
\Delta_{f}=\bigcap_{n \geqslant 1} \pi_{n *}\left\{\omega \in M^{1}\left(\Gamma_{n}\right):\left(f^{n}\right)^{*} \omega=d^{n} \cdot \pi_{n *} \omega\right\} .
$$

Each element of $\Delta_{f}$ is the projection of a solution to a pullback formula for each iterate of $f$, although we do not require any compatibility among these solutions. Linearity of the pullback and pushforward operators shows that $\Delta_{f}$ is a convex polyhedral set in the space $M^{1}(\Gamma)$. Note that $\Delta_{f}$ is nonempty: since $\omega_{f, \Gamma}=\omega_{f^{n}, \Gamma}$, the set $\Delta_{f}$ must contain the equilibrium $\Gamma$-measure $\omega_{f, \Gamma}$ (Lemma 4.4).

REMARK 4.9. The intersected sets that define $\Delta_{f}$ are typically not nested.

THEOREM 4.10. Let $f: \mathbf{P}^{1} \rightarrow \mathbf{P}^{1}$ be a rational function of degree $d \geqslant 2$, and let $\Gamma=\{\zeta\}$ be a singleton vertex set. Suppose that the Julia set of $f$ is not equal 
to $\{\zeta\}$. With the above notation, $\Delta_{f}$ is the convex hull of the equilibrium $\Gamma$ measure $\omega_{f, \Gamma}$ and at most one probability measure $\delta_{\mathcal{E}}$ supported on a classical exceptional orbit $\mathcal{E}$. Moreover, if $\Delta_{f} \neq\left\{\omega_{f, \Gamma}\right\}$, then $f^{n}(\zeta)$ converges to an exceptional orbit along the locus of total ramification for $f$.

REMARK 4.11. For our application to complex dynamics, it is sufficient to restrict to countably supported measures in the definition of $\Delta_{f}$. But the theorem shows that this hypothesis is unnecessary: an arbitrary $\Gamma$-measure satisfying all pullback formulas is countably supported.

REMARK 4.12. With a little more work, one can show that this result continues to hold when $k$ has positive characteristic provided that $\mathcal{O}_{f}(\zeta)$ does not converge to a wildly ramified exceptional orbit.

COROLlaRY 4.13. With the hypotheses of Theorem 4.10, no measure in $\Delta_{f}$ charges $\zeta$.

Proof. The hypothesis on the Julia set guarantees that $\zeta$ is not exceptional and that $\mu_{f}$ does not charge $\zeta$.

Proof of Theorem 4.10. Suppose that $f^{n}(\zeta)$ does not converge along the locus of total ramification to a classical exceptional periodic orbit for $f$. Let $U$ be any $\Gamma$-domain for $\Gamma=\{\zeta\}$. If $v \in \Delta_{f}$, Proposition 4.7 and the Surplus Estimate applied to $f^{n}$ and $U$ show that

$$
v(U) \geqslant \frac{s_{f^{n}}(U)}{d^{n}}=\mu_{f}(U)+o(1) .
$$

Since this is true for any $\Gamma$-disk $U$, and since $\mu_{f}$ is a probability measure with no support at $\zeta$, we conclude that $\nu(U)=\mu_{f}(U)$ for every $U \in \mathcal{S}(\Gamma)$.

Now suppose that $f^{n}(\zeta)$ converges along the locus of total ramification to the orbit of a classical exceptional point. Without loss of generality, we may assume that the exceptional point is fixed by replacing $f$ with $f^{2}$. After conjugating the exceptional fixed point to $\infty$, we may assume that $f$ is a polynomial. As in the proof of Proposition 4.7, we find that $f^{n}(\zeta)$ converges to $\infty$ along the segment $\left(\zeta_{0, R}, \infty\right)$ for some $R>1$, and $\zeta$ lies above the entire Julia set.

Suppose that $U$ is a $\Gamma$-domain that meets the Julia set. Then $f(U)$ contains the entire Julia set, and the standard pullback formula $f^{*} \mu_{f}=d \cdot \mu_{f}$ on $\mathbf{P}^{1}$ shows that

$$
d \cdot \mu_{f}(U)=m_{f}(U) \cdot \mu_{f}(f(U))=m_{f}(U) \quad \Rightarrow \quad \mu_{f}(U)=\frac{m_{f}(U)}{d} .
$$

In particular, only finitely many $\Gamma$-disks may meet the Julia set. 
Fix any $v \in \Delta_{f}$. Write $U_{\infty}$ for the unique $\Gamma$-domain containing infinity; write $U_{1}, \ldots, U_{r}$ for the $\Gamma$-domains that meet the Julia set; write $U_{0}$ for the union of the remaining elements of $\mathcal{S}(\Gamma)$. Note that since we are in case (2) of Proposition 4.7, the surplus multiplicity satisfies $s_{f^{n}}(U)=0$ for all $n \geqslant 1$ and $U \in \mathcal{S}(\Gamma)$. Furthermore, we observe that $f\left(U_{\infty}\right) \subset U_{\infty}$ and $m_{f^{n}}\left(U_{\infty}\right)=d^{n}$, and that $f^{n}$ maps $U_{0}$ onto $f^{n}\left(U_{0}\right) \subset U_{\infty}$ in everywhere $d^{n}$-to-one fashion.

First we show that $v\left(U_{0}\right)=0$. For each $n \geqslant 1$, there exists $v_{n} \in M^{1}\left(\Gamma_{n}\right)$ such that $\left(f^{n}\right)^{*} v_{n}=d^{n} \cdot \pi_{n *} v_{n}=d^{n} \cdot v$. Then

$$
d^{n} \cdot \pi_{n *} v_{n}\left(U_{\infty}\right)=\left(f^{n}\right)^{*} v_{n}\left(U_{\infty}\right)=\int\left(f^{n}\right)_{*} \chi_{U_{\infty}} v_{n}=d^{n} \cdot v_{n}\left(f^{n}\left(U_{\infty}\right)\right) .
$$

Thus $v\left(U_{\infty}\right)=v_{n}\left(f^{n}\left(U_{\infty}\right)\right)$ for any $n \geqslant 1$. Write $A$ for the annulus with boundary points $\zeta$ and $f^{n}(\zeta)$. By the definition of the pushforward, we see that

$$
v\left(U_{\infty}\right)=\pi_{n *} v_{n}\left(U_{\infty}\right)=v_{n}\left(f^{n}\left(U_{\infty}\right)\right)+v_{n}\left(f^{n}\left(U_{0}\right)\right)+v_{n}(A) .
$$

Therefore, $v_{n}(A)=v_{n}\left(f^{n}\left(U_{0}\right)\right)=0$. But the calculation (4.6.2) applies equally well to $U_{0}$, showing that $v\left(U_{0}\right)=v_{n}\left(f^{n}\left(U_{0}\right)\right)$, and so we conclude that $v\left(U_{0}\right)=0$.

Next we observe that for $i=1, \ldots, r$, we have

$$
d^{n} \cdot \pi_{n *} v_{n}\left(U_{i}\right)=\left(f^{n}\right)^{*} v_{n}\left(U_{i}\right)=\int\left(f^{n}\right)_{*} \chi_{U_{i}} v_{n}=m_{f^{n}}\left(U_{i}\right) \cdot v_{n}\left(f^{n}\left(U_{i}\right)\right) .
$$

From (4.6.1), we see that

$$
\mu_{f}\left(U_{i}\right)=\frac{m_{f}\left(U_{i}\right)}{d}=\frac{m_{f^{n}}\left(U_{i}\right)}{d^{n}}, \quad n \geqslant 1, \quad i=1, \ldots, r .
$$

Combining the last two displayed equations gives

$$
v\left(U_{i}\right)=\pi_{n *} v_{n}\left(U_{i}\right)=\mu_{f}\left(U_{i}\right) v_{n}\left(f^{n}\left(U_{i}\right)\right) .
$$

The quantity $a:=v_{n}\left(f^{n}\left(U_{i}\right)\right)$ is independent of $n$ and $i$ since $\mu_{f}\left(U_{i}\right)>0$ for $i=1, \ldots, r$ and $f^{n}\left(U_{1}\right)=\cdots=f^{n}\left(U_{r}\right)$. Setting $b=v\left(U_{\infty}\right)$, we have proved that $v=a \cdot \omega_{f, \Gamma}+b \cdot \delta_{\infty}$.

\section{A transfer principle}

In this section, we complete the proof of Theorem B. We explain the transfer of solutions of the pullback formula for the dynamics of our complex surface map $F: X \rightarrow Y$ to $\Gamma$-measure solutions of the pullback formula for a related function $f: \mathbf{P}^{1} \rightarrow \mathbf{P}^{1}$, and vice versa.

In all of the proofs, we must deal with two subcases: $Y=X$ and $Y \neq X$. We encourage the reader to think only of the former case on a first reading. As 
a further aid, we give a coarse sketch of the proof when $Y=X$ in the next subsection. Afterwards, we give a more general and rigorous description of the transfer process.

5.1. Outline of the transfer process when $\boldsymbol{Y}=\boldsymbol{X}$. As discussed in Section 3, we have a meromorphic one-parameter family of complex rational functions $f_{t}$, from which we define a rational self-map of the fibered surface $X=\mathbb{D} \times \mathbb{P}^{1}$ given by

$$
F: X \rightarrow X \quad F(t, z)=\left(t, f_{t}(z)\right) .
$$

The map $F$ is nonconstant on the central fiber $X_{0}=\{0\} \times \mathbb{P}^{1}$ if and only if the reduction of $f_{0}$ is nonconstant; we assume that these equivalent conditions hold.

The field $\mathbb{C}((t))$ of formal Laurent series in the variable $t$ has a natural nonArchimedean absolute value on it that measures the order of vanishing:

$$
\left|\sum_{n \geqslant N} a_{n} t^{n}\right|=\exp (-N) \quad\left(a_{N} \neq 0\right) .
$$

By viewing the parameter $t$ as a formal variable, we may identify the family $f_{t}$ with a single non-Archimedean rational function $f$ defined over the field $\mathbb{C}((t))$.

Recall that the Berkovich projective line $\mathbf{P}^{1}$ defined over $\mathbb{C}((t))$ has a natural tree structure with a canonical root $\zeta$, called the Gauss point. More is true: the branches of $\mathbf{P}^{1}$ at the Gauss point $\zeta$ are in canonical bijection with points of the Riemann sphere $\mathbb{P}^{1}(\mathbb{C})$. If we use the vertex set $\Gamma=\{\zeta\}$ on $\mathbf{P}^{1}$, then this bijection tells us how to relate Borel measures on $\mathbb{P}^{1}(\mathbb{C})$ with $\Gamma$-measures on $\mathbf{P}^{1}$. Namely, the point measure at $x \in \mathbb{P}^{1}(\mathbb{C})$ corresponds to the $\Gamma$-measure with all of its mass on the corresponding branch $U_{x}$, which is a $\Gamma$-disk in the sense of Section 4 . This correspondence extends by linearity to any purely atomic Borel measure on the Riemann sphere and any countably supported $\Gamma$-measure that does not charge the Gauss point. (Borel measures constructed in this way will be called residual measures.) Finally, a Borel measure $\mu$ of mass $M$ with no atom on $\mathbb{P}^{1}(\mathbb{C})$ gives rise to the $\Gamma$-measure $M \cdot \delta_{\zeta}$ on $\mathbf{P}^{1}$, but evidently this part of the correspondence has no inverse.

The Transfer Principle (Proposition 5.1) will show that this correspondence preserves solutions to the pullback equations $F^{*} \mu=d \cdot \mu$ for Borel measures on $X_{0}$ and $f^{*} \omega=d \cdot \omega$ for $\Gamma$-measures on $\mathbf{P}^{1}$. The argument is essentially formal once one proves equality of the local multiplicities and surplus multiplicities in the two definitions of pullback. It turns out that, even in the complex setting, each is an algebraic quantity that may be deduced from the expression for $f_{0}$.

5.2. Reduction and the residual measures. Let $X \rightarrow \mathbb{D}$ be a proper fibered surface over a complex disk with generic fiber $\mathbb{P}_{\mathbb{C}}^{1}$. Assume that the fiber $X_{0}$ over 
the origin is reduced. Let $\mathbb{L}$ be the completion of an algebraic closure of $\mathbb{C}((t))$ endowed with the natural non-Archimedean absolute value, and write $\mathbb{L}^{\circ}$ for its valuation ring. We claim that $X$ gives rise, canonically, to a vertex set $\Gamma \subset \mathbf{P}^{1}$. The local ring of $\mathbb{D}$ at the origin is contained inside $\mathbb{L}^{\circ}$, and hence so is its completion. By completing along the central fiber $X_{0}$ and base extending to $\mathbb{L}^{\circ}$, we obtain an admissible formal scheme $\mathfrak{X}$ over $\mathbb{L}^{\circ}$ with generic fiber $\mathbf{P}^{1}=\mathbf{P}_{\mathbb{L}}^{1}$. Note that since $X_{0}$ is reduced, it may be identified with the special fiber $\mathfrak{X}_{s}$ as $\mathbb{C}$-schemes. Let

$$
\operatorname{red}_{X}: \mathbf{P}^{1} \rightarrow X_{0}
$$

be the surjective reduction map $[2,2.4 .4]$. Let $\eta_{1}, \ldots, \eta_{r}$ be the generic points of the irreducible components of the special fiber $X_{0}$. There exist unique type II points $\zeta_{1}, \ldots, \zeta_{r} \in \mathbf{P}^{1}$ such that $\operatorname{red}_{X}\left(\zeta_{i}\right)=\eta_{i}$ for $i=1, \ldots, r$. The desired vertex set is $\Gamma=\left\{\zeta_{1}, \ldots, \zeta_{r}\right\}$.

For each closed point $x \in X_{0}$, the formal fiber $\operatorname{red}_{X}^{-1}(x)$ is a $\Gamma$-domain, as defined in Section 4.2. The association $x \mapsto \operatorname{red}_{X}^{-1}(x)$ induces a bijection between points of the scheme $X_{0}$ and elements of $\mathcal{S}(\Gamma)$. We obtain a projection of measures,

$$
\operatorname{red}_{X}^{*}: M^{1}\left(X_{0}\right) \rightarrow M^{1}(\Gamma),
$$

where $M^{1}\left(X_{0}\right)$ is the space of Borel probability measures on $X_{0}(\mathbb{C})$ (with its analytic topology) and $M^{1}(\Gamma)$ is the space of positive $\Gamma$-measures of total mass 1 on $\mathbf{P}^{1}$, defined as follows. Given $\mu \in M^{1}\left(X_{0}\right)$, let $B=\left\{x \in X_{0}(\mathbb{C}): \mu(\{x\})>0\right\}$. The set $B$ is at most countable. Write $\eta_{1}, \ldots, \eta_{r}$ for the generic points of the irreducible components $C_{1}, \ldots, C_{r}$ of $X_{0}$. Define $\omega=\operatorname{red}_{X}^{*}(\mu)$ by

$$
\omega\left(\operatorname{red}_{X}^{-1}(x)\right):= \begin{cases}\mu(x) & \text { if } x \in X_{0}(\mathbb{C}) \\ \mu\left(C_{i} \backslash B\right) & \text { if } x=\eta_{i} \text { for some } i=1, \ldots, r .\end{cases}
$$

Evidently, $\omega\left(\mathbf{P}^{1}\right)=\mu\left(X_{0}\right)=1$.

Now let $M^{1}(\Gamma)^{\dagger} \subset M^{1}(\Gamma)$ be the subset of $\Gamma$-measures that are countably supported on elements of $\mathcal{S}(\Gamma)$, and assign no mass to the elements of $\Gamma$. The reduction map $\operatorname{red}_{X}$ induces

$$
\operatorname{red}_{X *}: M^{1}(\Gamma)^{\dagger} \rightarrow M^{1}\left(X_{0}\right)
$$

as a partial inverse to $\operatorname{red}_{X}^{*}$. Explicitly, the residual measure $\mu=\operatorname{red}_{X *}(\omega) \in$ $M^{1}\left(X_{0}\right)$ is defined by

$$
\mu(\{x\}):=\omega\left(\operatorname{red}_{X}^{-1}(x)\right) \quad\left(x \in X_{0}(\mathbb{C})\right) .
$$

For each $\omega \in M^{1}(\Gamma)^{\dagger}$, the residual measure $\mu$ is an atomic probability measure on $X_{0}$. The terminology is explained by the case where $X_{0}$ is irreducible and $\Gamma=\left\{\zeta_{0,1}\right\}$ is the Gauss point of $\mathbf{P}^{1}$; the mass of the residual measure at a closed point $x \in X_{0}(\mathbb{C})$ is precisely the volume of the residue class $\operatorname{red}_{X}^{-1}(x) \subset \mathbf{P}^{1}$. 
5.3. Compatibility of pullbacks. Let $f_{t}$ be a one-parameter family of dynamical systems of degree $d \geqslant 2$ with $t$ varying holomorphically in a small punctured disk $\mathbb{D}^{*}$ and extending meromorphically over the puncture. As in Section 3, we let $X=\mathbb{D} \times \mathbb{P}^{1}(\mathbb{C})$ and write $\pi: Y \rightarrow X$ for the minimal modification of $X$ along $X_{0}$ such that the induced rational map $F: X \rightarrow Y$ is not constant along $X_{0}$. The surfaces $X$ and $Y$ induce vertex sets $\Gamma=\{\zeta\}$ and $\Gamma^{\prime}=\{\zeta, f(\zeta)\}$ on $\mathbf{P}^{1}=\mathbf{P}_{\mathbb{L}}^{1}$, where $\mathbb{L}$ is the completion of an algebraic closure of $\mathbb{C}((t))$ endowed with the natural non-Archimedean absolute value, and the family $f_{t}$ defines $f: \mathbf{P}^{1} \rightarrow \mathbf{P}^{1}$. The pullback $F^{*}$ from measures on $Y_{0}$ to measures on $X_{0}$ is given by the formula (3.3.1) or (3.3.2), depending on whether or not $f$ fixes $\zeta$.

Proposition 5.1 (Transfer Principle). Let $F: X \rightarrow Y, f: \mathbf{P}^{1} \rightarrow \mathbf{P}^{1}, \Gamma$, and $\Gamma^{\prime}$ be as above. The following conclusions hold.

(1) If $\mu$ is a measure on the central fiber $Y_{0}$ such that $F^{*} \mu=d \cdot \pi_{*} \mu$, then $\omega=\operatorname{red}_{Y}^{*} \mu$ is a $\Gamma^{\prime}$-measure satisfying $f^{*} \omega=d \cdot \pi_{*} \omega$.

(2) If $\omega$ is a countably supported $\Gamma^{\prime}$ probability measure satisfying $\omega\left(\Gamma^{\prime}\right)=0$ and $f^{*} \omega=d \cdot \pi_{*} \omega$, then the residual measure $\mu=\operatorname{red}_{Y *}(\omega)$ satisfies $F^{*} \mu=$ $d \cdot \pi_{*} \mu$.

Proof. We begin by comparing the notions of multiplicity defined for $F$ (on $X_{0}$ ) and for $f$ (on $\mathbf{P}^{1}$ ). Lemma 2.1 gives a meromorphic family of Möbius transformations $A_{t} \in \mathrm{PGL}_{2}(\mathbb{C})$ for $t \in \mathbb{D}$, holomorphic away from $t=0$, such that $A_{t} \circ f_{t}$ converges as $t \rightarrow 0$ to $\Phi \in \overline{\mathrm{Rat}}_{d}$ with nonconstant reduction $\phi$. On one hand, this implies that $\phi$ describes the meromorphic map $F$ from the fiber $X_{0}$ onto its image component $E$ in $Y_{0}$ (or $C$ if $X=Y$ ). Evidently the local degree $m(x)$ for each point of $X_{0}$ may be read off algebraically as the order of vanishing of $\phi(z)-\phi(x)$ at $x$. On the other hand, we may view $A_{t}$ as an element $A \in \mathrm{PGL}_{2}(\mathbb{C}((t)))$. In particular, $A \circ f$ has nonconstant reduction as a rational function on $\mathbf{P}^{1}$, and the reduction is equal to $\phi$. If $U_{x}$ is a $\Gamma$-disk with reduction $x \in X_{0}$, Rivera-Letelier's Algebraic Reduction Formula [1, Corollary 9.25] shows that the directional multiplicity $m_{f}\left(U_{x}\right)$ is equal to the order of vanishing of $\phi(z)-\phi(x)$ at $x$, and so we conclude that

$$
m(x)=m_{f}\left(U_{x}\right)
$$

From the description of the surplus multiplicity of the map $\Phi$ in (2.2.1) and the corresponding description of the surplus multiplicity in [8, Lemma 3.17], we also see that

$$
s(x)=s_{f}\left(U_{x}\right) .
$$


Finally, the Algebraic Reduction Formula shows that $\operatorname{deg}(\phi)=m_{f}(\zeta)$, where $\zeta$ is the unique vertex in $\Gamma$.

Since $\omega=\operatorname{red}_{Y}^{*}(\mu)$ is supported on countably many $\Gamma^{\prime}$-domains, to prove the first statement of the Transfer Principle it suffices to show that

$$
F^{*} \mu=d \cdot \pi_{*} \mu \Rightarrow f^{*} \omega(U)=d \cdot \pi_{*} \omega(U) \text { for every } U \in \mathcal{S}(\Gamma) .
$$

Under the hypotheses of the second statement of the Transfer Principle, we find that $\mu$ is countably supported. Thus it suffices to show that

$$
f^{*} \omega=d \cdot \pi_{*} \omega \Rightarrow F^{*} \mu(\{x\})=d \cdot \pi_{*} \mu(\{x\}) \text { for every closed point } x \in X_{0} .
$$

We now prove these statements.

Case $\boldsymbol{Y}=\boldsymbol{X}$. Let $x \in X_{0}$ be a closed point, and write $U_{x}=\operatorname{red}_{X}^{-1}(x) \in \mathcal{S}(\Gamma)$. Then

$$
\begin{aligned}
f^{*} \omega\left(U_{x}\right) & =\sum_{V \in S(\Gamma)} m_{V, U_{x}} \omega(V) \\
& =m_{\bar{f}\left(U_{x}\right), U_{x}} \omega\left(\bar{f}\left(U_{x}\right)\right)+\sum_{V \in \mathcal{S}(\Gamma)} s_{f}\left(U_{x}\right) \omega(V) \\
& =m(x) \mu(\{\phi(x)\})+s(x) \\
& =F^{*} \mu(\{x\}),
\end{aligned}
$$

while $d \cdot \omega\left(U_{x}\right)=d \cdot \mu(\{x\})$ by definition. This immediately implies equation (TP2).

To verify equation (TP1), it remains to consider the mass on $\Gamma=\Gamma^{\prime}$. Set $B=$ $\left\{x \in X_{0}: \mu(x)>0\right\}$. If $F^{*} \mu=d \cdot \mu$, then $F^{*} \mu$ has no atoms in $X_{0} \backslash B$. From the definition of $F^{*} \mu$ in (3.3.1), we see that $F^{*} \mu$ agrees with $\phi^{*} \mu$ on $X_{0} \backslash B$. Thus,

$$
\begin{aligned}
d \cdot \omega(\zeta)=d \cdot \mu\left(X_{0} \backslash B\right) & =F^{*} \mu\left(X_{0} \backslash B\right) \\
& =\phi^{*} \mu\left(X_{0} \backslash B\right)=\operatorname{deg}(\phi) \cdot \mu\left(X_{0} \backslash B\right) \\
& =m_{f}(\zeta) \omega(\zeta)=f^{*} \omega(\zeta) .
\end{aligned}
$$

This proves equation (TP1) for all $U$ in $\mathcal{S}(\Gamma)$.

Case $Y \neq X$. Recall that $Y_{0}=C \cup E$, where $C$ is the proper transform of $X_{0}$, and $E$ is the exceptional fiber of $\pi: Y \rightarrow X$. In Section 3.3, to define $F^{*} \mu$ we introduced the (continuous) projection $\pi_{E}: Y_{0} \rightarrow E$ that collapses $C$ to a point. 
Let $U_{x}$ be the $\Gamma$-disk corresponding to a closed point $x \in X_{0}$. Recall that we set $\varepsilon\left(V, U_{x}\right)=1$ or 0 depending on whether $\bar{f}\left(U_{x}\right)=V$ or not. We see that

$$
\begin{aligned}
f^{*} \omega\left(U_{x}\right) & =\sum_{V \in \mathcal{S}\left(\Gamma^{\prime}\right)} m_{V, U_{x}} \omega(V) \\
& =\sum_{V \in \mathcal{S}\left(\Gamma^{\prime}\right)}\left(s_{f}\left(U_{x}\right)+m_{f}\left(U_{x}\right) \varepsilon\left(V, U_{x}\right)\right) \omega(V) \\
& =s(x)+m(x)\left(\pi_{E *} \mu\right)(\{\phi(x)\}) \\
& =F^{*} \mu(\{x\}),
\end{aligned}
$$

while $\pi_{*} \omega\left(U_{x}\right)=\pi_{*} \mu(\{x\})$ is immediate. Evidently (TP2) follows, and (TP1) holds for all $\Gamma$-disks.

To verify (TP1), it remains to check the pullback relation for the mass on vertices. Let $B=\left\{y \in Y_{0}: \mu(\{y\})>0\right\}$, and set $B^{\prime}=\pi(B \cup E) \subset X_{0}$. Then

$$
d \cdot \pi_{*} \mu\left(X_{0} \backslash B^{\prime}\right)=d \cdot \mu\left(\pi^{-1}\left(X_{0} \backslash B^{\prime}\right)\right)=d \cdot \mu(C \backslash B) .
$$

If $F^{*} \mu=d \cdot \pi_{*} \mu$, there are no atoms of $F^{*} \mu$ outside $B^{\prime}$. From the definition of $F^{*} \mu$ in (3.3.2), the measure $F^{*} \mu$ must agree with the pullback of $\pi_{E *} \mu$ by $\phi$ on the set $X_{0} \backslash B^{\prime}$; therefore,

$$
F^{*} \mu\left(X_{0} \backslash B^{\prime}\right)=\phi^{*}\left(\pi_{E *} \mu\right)\left(X_{0} \backslash B^{\prime}\right)=\operatorname{deg}(\phi) \cdot \mu(E \backslash B) .
$$

Putting these observations together yields

$$
f^{*} \omega(\zeta)=m_{f}(\zeta) \omega(f(\zeta))=\operatorname{deg}(\phi) \mu(E \backslash B)=d \cdot \mu(C \backslash B)=d \cdot \pi_{*} \omega(\zeta),
$$

so (TP1) is verified for all $U$ in $\mathcal{S}(\Gamma)$.

5.4. Proof of Theorem B. We retain all of the notation from previous sections.

For each $n \geqslant 1$, let $F_{n}: X \rightarrow Y^{n}$ be the rational map of surfaces associated with the one-parameter family of $n$th iterates $f_{t}^{n}$, as constructed in Section 2, and write $\pi_{n}: Y^{n} \rightarrow X$ for the blowing-up morphism. Define

$$
\Delta_{0}=\bigcap_{n \geqslant 1} \pi_{n *}\left\{\mu \in M^{1}\left(Y_{0}^{n}\right): F_{n}^{*} \mu=d^{n} \cdot \pi_{n *} \mu\right\} \subset M^{1}\left(X_{0}\right) .
$$

Write $\omega_{f, \Gamma}$ for the equilibrium $\Gamma$-measure for $f: \mathbf{P}^{1} \rightarrow \mathbf{P}^{1}$ associated with $\Gamma=$ $\{\zeta\}$. Recall that $\Delta_{f}$ was defined in Section 4.6. 
THEOREM 5.2. Let $f_{t}$ be a meromorphic one-parameter family of rational functions of degree $d \geqslant 2$. Suppose that the family is not holomorphic at $t=0$; i.e., $\operatorname{deg}\left(f_{0}\right)<d$. The reduction map induces a bijection

$$
\operatorname{red}_{X}^{*}: \Delta_{0} \stackrel{\sim}{\rightarrow} \Delta_{f},
$$

with inverse given by the residual measure construction $\operatorname{red}_{X *}$.

Proof. No measure in $\Delta_{f}$ charges the vertex $\zeta \in \Gamma$, and every measure in $\Delta_{f}$ is countably supported (Theorem 4.10). The Transfer Principle (applied to all iterates of $f_{t}$ and $f$ ) shows that the maps

$$
\operatorname{red}_{X}^{*}: \Delta_{0} \rightarrow \Delta_{f} \quad \text { and } \quad \operatorname{red}_{X *}: \Delta_{f} \rightarrow \Delta_{0}
$$

are well defined. That they are inverse to one another follows from the definitions of $\operatorname{red}_{X}^{*}$ and $\operatorname{red}_{X *}$.

COROLLARY 5.3. With the setup of Theorem 5.2, $\Delta_{0}$ always contains the residual measure $\operatorname{red}_{X *}\left(\omega_{f, \Gamma}\right)$, and $\Delta_{0}$ is either a point or a segment in the space of all probability measures. In the latter case, there exists a point mass $\delta_{p_{0}} \in \Delta_{0}$ and a one-parameter family of exceptional periodic points $p_{t}$ for $f_{t}$ such that $f_{0}$ is constant with value $p_{0}$, and $p_{0}$ is not an indeterminacy point for the rational map $F: X \rightarrow X$.

Proof. Theorem 5.2 allows us to transfer the statements about $\Delta_{0}$ to $\Delta_{f}$. The first statement is immediate from Theorem 4.10. If $\Delta_{f} \neq\left\{\omega_{f, \Gamma}\right\}$, then $f^{n}(\zeta)$ converges along the locus of total ramification to a classical exceptional orbit $\mathcal{E}$. Replacing $f$ and $f_{t}$ with their second iterates if necessary, we may assume that $\mathcal{E}=\{p\}$ is a single point. Now $p \in \mathbb{P}^{1}(\mathbb{C}((t)))$ by completeness. A priori, this gives a formal one-parameter family $p_{t}$ with complex coefficients. Since $f_{t}\left(p_{t}\right)=p_{t}$ and $\frac{d f_{t}}{d z}\left(p_{t}\right) \equiv 0$, the implicit function theorem shows that $p_{t}$ is a meromorphic oneparameter family in a small disk about $t=0$. That is, $p=p_{t}$ is a one-parameter family of exceptional fixed points for the family $f_{t}$. Since $f^{n}(\zeta)$ converges to $p$, and since $p$ is a superattracting fixed point for $f$, it follows that $f_{0}$ is constant with value equal to $p_{0}$. If $U$ is the open $\Gamma$-disk containing $p$, then $f(U) \subsetneq U$. In particular, this shows that $s_{f}(U)=s\left(p_{0}\right)=0$, so $p_{0}$ is not an indeterminacy point for the rational map $F$.

We are now ready to prove the second main result of the article. With the terminology that we have set up in the preceding sections, our goal is to show that the family of measures of maximal entropy $\left\{\mu_{t}: t \in \mathbb{D}^{*}\right\}$ converges weakly to the residual measure $\operatorname{red}_{X *}\left(\omega_{f, \Gamma}\right)$ as $t \rightarrow 0$, where $\Gamma=\{\zeta\}$ is the Gauss point of $\mathbf{P}^{1}$. 
Proof of Theorem $B$. Let $\mu^{1}$ be any weak limit of the family $\mu_{t}$ of maximal measures as $t \rightarrow 0$ on the surface $Y^{1}$. Fix a subsequence $\left(t_{\ell}\right)_{\ell \geqslant 1}$ such that $t_{\ell} \rightarrow 0$ and $\mu_{t_{\ell}} \rightarrow \mu^{1}$ weakly on $Y^{1}$. Set $\mu_{0}=\pi_{1 *} \mu^{1}$; then $\mu_{t_{\ell}} \rightarrow \mu_{0}$ weakly on $X$.

For each $n \geqslant 2$, let $\mu^{n}$ be a weak limit of the sequence $\left(\mu_{t_{\ell}}\right)$ on the surface $Y^{n}$. Note that $\mu_{0}=\pi_{n *} \mu^{n}$ by construction. Moreover, we have $F_{n}^{*} \mu^{n}=d^{n} \cdot \pi_{n *} \mu^{n}$ for all $n \geqslant 1$ (Theorem 3.5). Hence $\mu_{0} \in \Delta_{0}$.

It remains to prove that $\mu_{0}=\operatorname{red}_{X *}\left(\omega_{f, \Gamma}\right)$, the residual measure associated with $\omega_{f}$ and the vertex set $\Gamma$. This follows immediately from the preceding corollary unless there exists a family of exceptional periodic points $p_{t}$ for $f_{t}$, the reduction of $f_{0}$ is equal to the constant $p_{0}$, and $p_{0}$ is not indeterminate for the rational map $F$. In that case, $\mu_{0}=a \cdot \operatorname{red}_{X *}\left(\omega_{f, \Gamma}\right)+b \cdot \operatorname{red}_{X *}\left(\delta_{\mathcal{E}}\right)$, for some $a, b \geqslant 0$, where $p_{0} \in \operatorname{supp}\left(\operatorname{red}_{X *}\left(\delta_{\mathcal{E}}\right)\right)$. We must prove that $b=0$.

Since $p_{0}$ is not indeterminate, by continuity there exists a neighborhood $N$ of $p_{0}$ such that $f_{t}(N) \subset N$ for all $t$ sufficiently close to zero. Hence, $N$ is contained in the Fatou set of $f_{t}$, and $\mu_{t}$ assigns no mass to $N$. By weak continuity, $\mu_{0}(N)=0$. That is, $b=0$ and $\mu_{0}=\operatorname{red}_{X *}\left(\omega_{f, \Gamma}\right)$ as desired.

\section{Acknowledgements}

We are grateful for the opportunities that allowed these ideas to germinate: the 2010 Bellairs Workshop in Number Theory funded by the CRM in Montreal, and the Spring 2012 semester on Complex and Arithmetic Dynamics at ICERM. We would like to thank Charles Favre, Mattias Jonsson, Jan Kiwi, and Juan RiveraLetelier for helpful discussions, and we further thank Jonsson for inviting us to speak about this work at the December 2012 RTG workshop at the University of Michigan. Finally, we thank the anonymous referee for useful suggestions for improving the presentation.

\section{References}

[1] M. Baker and R. Rumely, Potential Theory and Dynamics on the Berkovich Projective Line, Mathematical Surveys and Monographs, 159 (American Mathematical Society, Providence, RI, 2010).

[2] V. G. Berkovich, Spectral Theory and Analytic Geometry Over Non-Archimedean Fields, Mathematical Surveys and Monographs, 33 (American Mathematical Society, Providence, RI, 1990).

[3] A. Bonifant, J. Kiwi and J. Milnor, 'Cubic polynomial maps with periodic critical orbit. II. Escape regions', Conform. Geom. Dyn. 14 (2010), 68-112.

[4] L. DeMarco, 'Iteration at the boundary of the space of rational maps', Duke Math. J. 130 (1) (2005), 169-197.

[5] L. DeMarco, 'The moduli space of quadratic rational maps', J. Amer. Math. Soc. 20 (2) (2007), $321-355$. 
[6] L. DeMarco and X. Faber, 'Degenerations of complex dynamical systems II: Analytic and algebraic stability'. Preprint, arXiv:1309.7103 [math.DS], 2013.

[7] L. G. DeMarco and C. T. McMullen, 'Trees and the dynamics of polynomials', Ann. Sci. Éc. Norm. Supér. (4) 41 (3) (2008), 337-382.

[8] X. Faber, 'Topology and geometry of the Berkovich ramification locus for rational functions, I', Manuscripta Math. 142 (3-4) (2013), 439-474.

[9] X. Faber, 'Topology and geometry of the Berkovich ramification locus for rational functions, II', Math. Ann. 356 (3) (2013), 819-844.

[10] C. Favre, Personal communication.

[11] C. Favre and J. Rivera-Letelier, 'Théorie ergodique des fractions rationnelles sur un corps ultramétrique', Proc. Lond. Math. Soc. (3) 100 (1) (2010), 116-154.

[12] A. Freire, A. Lopes and R. Mañé, 'An invariant measure for rational maps', Bol. Soc. Brasil. Mat. 14 (1) (1983), 45-62.

[13] J. Kiwi, 'Puiseux series polynomial dynamics and iteration of complex cubic polynomials', Ann. Inst. Fourier (Grenoble) 56 (5) (2006), 1337-1404.

[14] J. Kiwi, 'Rescaling limits of complex rational maps'. arXiv:1211.3397 [math.DS]. Preprint, 2012.

[15] M. J. Ljubich, 'Entropy properties of rational endomorphisms of the Riemann sphere', Ergodic Theory Dynam. Systems 3 (3) (1983), 351-385.

[16] R. M. P. Sad and D. Sullivan, 'On the dynamics of rational maps', Ann. Sci. École Norm. Sup. (4) 16 (2) (1983), 193-217.

[17] R. Mañé, 'On the uniqueness of the maximizing measure for rational maps', Bol. Soc. Brasil. Mat. 14 (1) (1983), 27-43.

[18] R. Mañé, 'The Hausdorff dimension of invariant probabilities of rational maps', in Dynamical systems, Valparaiso 1986, Lecture Notes in Math., 1331 (Springer, Berlin, 1988), 86-117.

[19] J. R. Letelier, 'Dynamique des fonctions rationnelles sur des corps locaux', Astérisque 287 (xv) (2003), 147-230. Geometric methods in dynamics. II. 